DESY 00-170

hep-ph/0011323

November 2000

\title{
B PHYSICS AND CP VIOLATION
}

\author{
R. FLEISCHER \\ Deutsches Elektronen-Synchrotron DESY \\ Notkestr. 85, D-22607 Hamburg, Germany
}

\begin{abstract}
After an introduction to the Standard-Model description of $C P$ violation and a brief look at the present status of this phenomenon in the kaon system, a classification of non-leptonic $B$-decays is given and the formalism of $B_{d, s}^{0}-\overline{B_{d, s}^{0}}$ mixing is discussed. We then turn to the $B$-factory benchmark modes, $C P$ violation in charged $B$ decays, and the $B_{s}$-meson system. Finally, we focus both on $B \rightarrow \pi K$ decays, which play an important role to probe the CKM angle $\gamma$, and on the $B_{d} \rightarrow \pi^{+} \pi^{-}, B_{s} \rightarrow K^{+} K^{-}$ system, which allows an interesting determination of $\beta$ and $\gamma$.

(Invited lecture at NATO ASI 2000, Cascais, Portugal, 26 June - 7 July, 2000)
\end{abstract}

\section{Introduction}

The violation of the $C P$ symmetry, where $C$ and $P$ denote the chargeconjugation and parity-transformation operators, respectively, is one of the fundamental phenomena in particle physics. Although weak interactions are neither invariant under $P$, nor invariant under $C$, it was originally believed that the product $C P$ was preserved. Consider, for instance, the process

$$
\pi^{+} \rightarrow e^{+} \nu_{e} \stackrel{C}{\longrightarrow} \pi^{-} \rightarrow e^{-} \nu_{e}^{C} \stackrel{P}{\longrightarrow} \pi^{-} \rightarrow e^{-} \bar{\nu}_{e} .
$$

Here the left-handed $\nu_{e}^{C}$ state is not observed in nature; only after performing an additional parity transformation we obtain the right-handed electron antineutrino. In 1964, it was then found experimentally through the observation of $K_{\mathrm{L}} \rightarrow \pi^{+} \pi^{-}$decays that weak interactions are not invariant under $C P$ transformations [1]. So far, $C P$ violation has only been observed in the kaon system, and we still have few experimental insights into this phenomenon. However, the measurement of $C P$ asymmetries should also be "around the corner" in $B$ decays [2], which are currently explored in great detail at the $B$-factories. For a collection of basic references on $C P$ violation and $B$ physics, the reader is referred to Refs. $[3,4]$. 
Studies of $C P$-violating effects are very exciting, since physics beyond the Standard Model is usually associated with new sources for $C P$ violation. Important examples are non-minimal supersymmetry, left-right-symmetric models, models with extended Higgs sectors, and many other scenarios for "new" physics [5]. In this context, it is also interesting to note that the evidence for neutrino masses we got during the recent years points towards physics beyond the Standard Model [6], raising the question of $C P$ violation in the neutrino sector [7], which may be studied in the more distant future at $\nu$-factories. In cosmology, $C P$ violation plays also a crucial role: one of the necessary conditions to generate the matter-antimatter asymmetry of our Universe is - in addition to baryon number violation and deviations from thermal equilibrium - that the elementary interactions have to violate $C P$ (and $C$ ) $[8,9]$. Recent model calculations indicate, however, that the $C P$ violation present in the Standard Model is too small to generate the observed matter-antimatter asymmetry of $\mathcal{O}\left(10^{-10}\right)$ [10].

Concerning quantitative tests of the Standard-Model description of $C P$ violation, the $B$-meson system is particularly promising. In the search for new physics, it is crucial to have $C P$-violating $B$-decay processes available that can be analysed reliably within the framework of the Standard Model, which will be the major topic of these lectures. Presently, we are at the beginning of the $B$-factory era in particle physics, and in the summer of 2000, the BaBar (SLAC) and Belle (KEK) collaborations have already reported their first results. Moreover, HERA-B (DESY) has seen first events, CLEO-III (Cornell) has started taking data, and run II of the Tevatron (Fermilab) will follow next spring. A lot of interesting physics will also be left for "second-generation" $B$-experiments at hadron machines, $\mathrm{LHCb}$ (CERN) and BTeV (Fermilab). Detailed studies of the $B$-physics potentials of BaBar, run II of the Tevatron, and the LHC can be found in Ref. [11].

The outline of these lectures is as follows: in Section 2, we discuss the Standard-Model description of $C P$ violation. After a brief look at the present status of $C P$ violation in the kaon system in Section 3, we turn to the $B$ system in Section 4 by giving a classification of non-leptonic $B$ decays and introducing low-energy effective Hamiltonians. A key element for $C P$ violation in the $B$ system - the formalism of $B_{d, s}^{0}-\overline{B_{d, s}^{0}}$ mixing - is presented in Section 5, and is applied to important $B$-factory benchmark modes in Section 6 . We then turn to $C P$ violation in charged $B$ decays in Section 7 , and discuss the $B_{s}$-meson system - the "El Dorado" for hadron machines - in Section 8. The remainder of these lectures is devoted to two more recent developments: the phenomenology of $B \rightarrow \pi K$ decays, which is the topic of Section 9 , and the $B_{d} \rightarrow \pi^{+} \pi^{-}, B_{s} \rightarrow K^{+} K^{-}$system, which is discussed Section 10. Before concluding in Section 12, we make a few comments on other interesting rare $B$ decays in Section 11. 


\section{The Standard-Model Description of CP Violation}

Within the Standard Model of electroweak interactions [12], $C P$ violation is closely related to the Cabibbo-Kobayashi-Maskawa (CKM) matrix [13, 14], connecting the electroweak eigenstates $\left(d^{\prime}, s^{\prime}, b^{\prime}\right)$ of the down, strange and bottom quarks with their mass eigenstates $(d, s, b)$ through the following unitary transformation:

$$
\left(\begin{array}{c}
d^{\prime} \\
s^{\prime} \\
b^{\prime}
\end{array}\right)=\left(\begin{array}{ccc}
V_{u d} & V_{u s} & V_{u b} \\
V_{c d} & V_{c s} & V_{c b} \\
V_{t d} & V_{t s} & V_{t b}
\end{array}\right) \cdot\left(\begin{array}{c}
d \\
s \\
b
\end{array}\right) \equiv \hat{V}_{\mathrm{CKM}} \cdot\left(\begin{array}{c}
d \\
s \\
b
\end{array}\right) .
$$

The elements of the CKM matrix describe charged-current couplings, as can be seen easily by expressing the non-leptonic charged-current interaction Lagrangian in terms of the mass eigenstates appearing in (2):

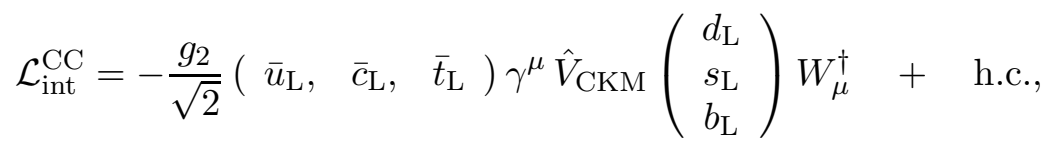

where the gauge coupling $g_{2}$ is related to the gauge group $S U(2)_{\mathrm{L}}$, and the $W_{\mu}^{(\dagger)}$ field corresponds to the charged $W$-bosons.

\subsection{PARAMETRIZATIONS OF THE CKM MATRIX}

The phase structure of the CKM matrix is not unique, as we may perform the following phase transformations:

$$
V_{U D} \rightarrow \exp \left(i \xi_{U}\right) V_{U D} \exp \left(-i \xi_{D}\right)
$$

which are related to redefinitions of the up- and down-type quark fields:

$$
U \rightarrow \exp \left(i \xi_{U}\right) U, \quad D \rightarrow \exp \left(i \xi_{D}\right) D .
$$

Using these transformations, it can be shown that the general $N$-generation quark-mixing-matrix is described by $(N-1)^{2}$ parameters, which consist of $N(N-1) / 2$ Euler-type angles, and $(N-1)(N-2) / 2$ complex phases. In the two-generation case [13], we arrive therefore at

$$
\hat{V}_{\mathrm{C}}=\left(\begin{array}{cc}
\cos \theta_{\mathrm{C}} & \sin \theta_{\mathrm{C}} \\
-\sin \theta_{\mathrm{C}} & \cos \theta_{\mathrm{C}}
\end{array}\right)
$$

where $\sin \theta_{\mathrm{C}}=0.22$ can be determined from $K \rightarrow \pi e^{+} \nu_{e}$ decays.

In the case of three generations, three Euler-type angles and a single complex phase are needed to parametrize the CKM matrix. This complex 


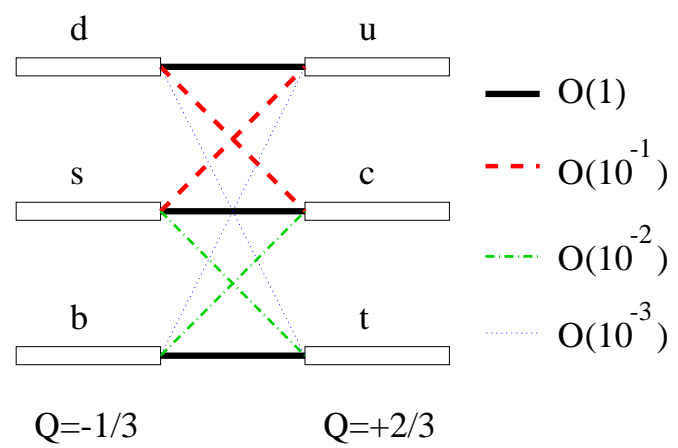

Figure 1. Hierarchy of the quark transitions mediated through charged currents.

phase allows us to accommodate $C P$ violation in the Standard Model, as was pointed out by Kobayashi and Maskawa in 1973 [14]. In the "standard parametrization", the three-generation CKM matrix takes the form

$$
\left(\begin{array}{ccc}
c_{12} c_{13} & s_{12} c_{13} & s_{13} e^{-i \delta_{13}} \\
-s_{12} c_{23}-c_{12} s_{23} s_{13} e^{i \delta_{13}} & c_{12} c_{23}-s_{12} s_{23} s_{13} e^{i \delta_{13}} & s_{23} c_{13} \\
s_{12} s_{23}-c_{12} c_{23} s_{13} e^{i \delta_{13}} & -c_{12} s_{23}-s_{12} c_{23} s_{13} e^{i \delta_{13}} & c_{23} c_{13}
\end{array}\right),
$$

where $c_{i j}=\cos \theta_{i j}$ and $s_{i j}=\sin \theta_{i j}$. Another interesting parametrization of the CKM matrix was proposed by Fritzsch and Xing [15], which is based on the hierarchical structure of the quark mass spectrum.

In Fig. 1, the hierarchy of the strengths of the quark transitions mediated through charged-current interactions is illustrated. In the standard parametrization (7), it is reflected by

$$
s_{12}=0.22 \gg s_{23}=\mathcal{O}\left(10^{-2}\right) \gg s_{13}=\mathcal{O}\left(10^{-3}\right) .
$$

If we introduce new parameters $\lambda, A, \rho$ and $\eta$ by imposing the relations

$$
s_{12} \equiv \lambda=0.22, \quad s_{23} \equiv A \lambda^{2}, \quad s_{13} e^{-i \delta} \equiv A \lambda^{3}(\rho-i \eta),
$$

and go back to the standard parametrization (7), we arrive at

$$
\hat{V}_{\mathrm{CKM}}=\left(\begin{array}{ccc}
1-\frac{1}{2} \lambda^{2} & \lambda & A \lambda^{3}(\rho-i \eta) \\
-\lambda & 1-\frac{1}{2} \lambda^{2} & A \lambda^{2} \\
A \lambda^{3}(1-\rho-i \eta) & -A \lambda^{2} & 1
\end{array}\right)+\mathcal{O}\left(\lambda^{4}\right) .
$$

This is the "Wolfenstein parametrization" of the CKM matrix [16]. It corresponds to an expansion in powers of the small quantity $\lambda=0.22$, and is very useful for phenomenological applications. A detailed discussion of the next-to-leading order terms in $\lambda$ can be found in Ref. [17]. 


\subsection{FURTHER REQUIREMENTS FOR CP VIOLATION}

As we have just seen, three generations are necessary to accommodate $C P$ violation in the Standard Model. However, still more conditions have to be satisfied. They can be summarized as follows:

$$
\begin{aligned}
& \left(m_{t}^{2}-m_{c}^{2}\right)\left(m_{t}^{2}-m_{u}^{2}\right)\left(m_{c}^{2}-m_{u}^{2}\right) \\
& \quad \times\left(m_{b}^{2}-m_{s}^{2}\right)\left(m_{b}^{2}-m_{d}^{2}\right)\left(m_{s}^{2}-m_{d}^{2}\right) \times J_{\mathrm{CP}} \neq 0,
\end{aligned}
$$

where

$$
J_{\mathrm{CP}}= \pm \operatorname{Im}\left(V_{i \alpha} V_{j \beta} V_{i \beta}^{*} V_{j \alpha}^{*}\right) \quad(i \neq j, \alpha \neq \beta) .
$$

The "Jarlskog Parameter" $J_{\mathrm{CP}}$ represents a measure of the "strength" of $C P$ violation within the Standard Model [18]. Using the Wolfenstein parametrization, we obtain

$$
J_{\mathrm{CP}}=\lambda^{6} A^{2} \eta=\mathcal{O}\left(10^{-5}\right) .
$$

Consequently, $C P$ violation is a small effect in the Standard Model. However, typically new complex couplings are present in scenarios for new physics, yielding additional sources for $C P$ violation.

\subsection{THE UNITARITY TRIANGLES OF THE CKM MATRIX}

Concerning tests of the CKM picture of $C P$ violation, the central targets are the unitarity triangles of the CKM matrix. The unitarity of the CKM matrix, which is described by

$$
\hat{V}_{\mathrm{CKM}}^{\dagger} \cdot \hat{V}_{\mathrm{CKM}}=\hat{1}=\hat{V}_{\mathrm{CKM}} \cdot \hat{V}_{\mathrm{CKM}}^{\dagger},
$$

leads to a set of 12 equations, consisting of 6 normalization relations and 6 orthogonality relations. The latter can be represented as 6 triangles in the complex plane, all having the same area, $2 A_{\Delta}=\left|J_{\mathrm{CP}}\right|[19]$. However, in only two of them, all three sides are of comparable magnitude $\mathcal{O}\left(\lambda^{3}\right)$, while in the remaining ones, one side is suppressed relative to the others by $\mathcal{O}\left(\lambda^{2}\right)$ or $\mathcal{O}\left(\lambda^{4}\right)$. The orthogonality relations describing the non-squashed triangles are given by

$$
\begin{array}{llll}
V_{u d} V_{u b}^{*}+V_{c d} V_{c b}^{*}+V_{t d} V_{t b}^{*}= & 0 & {[1 \text { st and 3rd column }]} \\
V_{u b}^{*} V_{t b}+V_{u s}^{*} V_{t s}+V_{u d}^{*} V_{t d}= & 0 & {[1 \text { st and 3rd row }]}
\end{array}
$$

At leading order in $\lambda$, these relations agree with each other, and yield

$$
(\rho+i \eta) A \lambda^{3}+\left(-A \lambda^{3}\right)+(1-\rho-i \eta) A \lambda^{3}=0 .
$$



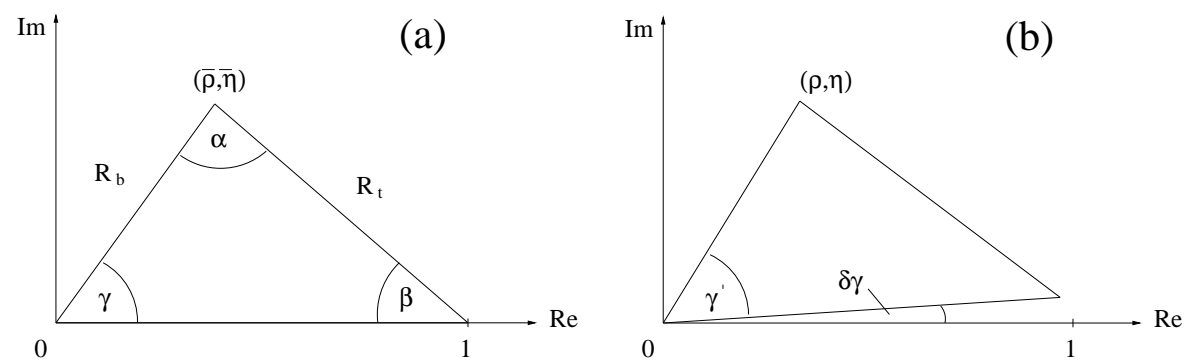

Figure 2. The two non-squashed unitarity triangles of the CKM matrix: (a) and (b) correspond to the orthogonality relations (15) and (16), respectively.

Consequently, they describe the same triangle in the $\rho-\eta$ plane $^{1}$, which is usually referred to as "the" unitarity triangle of the CKM matrix [20]. However, in the era of second-generation $B$ experiments, the experimental accuracy will be so tremendous that we will also have to take into account the next-to-leading order terms of the Wolfenstein expansion, and will have to distinguish between the unitarity triangles described by (15) and (16). They are illustrated in Fig. 2, where $\bar{\rho}$ and $\bar{\eta}$ are related to the Wolfenstein parameters $\rho$ and $\eta$ through [17]

$$
\bar{\rho} \equiv\left(1-\lambda^{2} / 2\right) \rho, \quad \bar{\eta} \equiv\left(1-\lambda^{2} / 2\right) \eta
$$

Note that $\gamma=\gamma^{\prime}+\delta \gamma$. The sides $R_{b}$ and $R_{t}$ of the unitarity triangle shown in Fig. 2 (a) are given as follows:

$$
\begin{aligned}
& R_{b}=\left(1-\frac{\lambda^{2}}{2}\right) \frac{1}{\lambda}\left|\frac{V_{u b}}{V_{c b}}\right|=\sqrt{\bar{\rho}^{2}+\bar{\eta}^{2}}=0.41 \pm 0.07 \\
& R_{t}=\frac{1}{\lambda}\left|\frac{V_{t d}}{V_{c b}}\right|=\sqrt{(1-\bar{\rho})^{2}+\bar{\eta}^{2}}=\mathcal{O}(1)
\end{aligned}
$$

and will show up at several places throughout these lectures.

\subsection{TOWARDS AN ALLOWED RANGE IN THE $\bar{\rho}-\bar{\eta}$ PLANE}

The parameter $R_{b}$ introduced in (19), i.e. the ratio $\left|V_{u b}\right| /\left|V_{c b}\right|$, can be determined through semi-leptonic $b \rightarrow u$ and $b \rightarrow c$ decays. It fixes a circle in the $\bar{\rho}-\bar{\eta}$ plane around $(0,0)$ with radius $R_{b}$. The second side $R_{t}$ of the unitarity triangle shown in Fig. 2 (a) can be determined through $B_{d, s}^{0}-\overline{B_{d, s}^{0}}$ mixing. It fixes another circle in the $\bar{\rho}-\bar{\eta}$ plane, which is centered at $(1,0)$

\footnotetext{
${ }^{1}$ Usually, the triangle relation (17) is divided by the overall normalization $A \lambda^{3}$.
} 


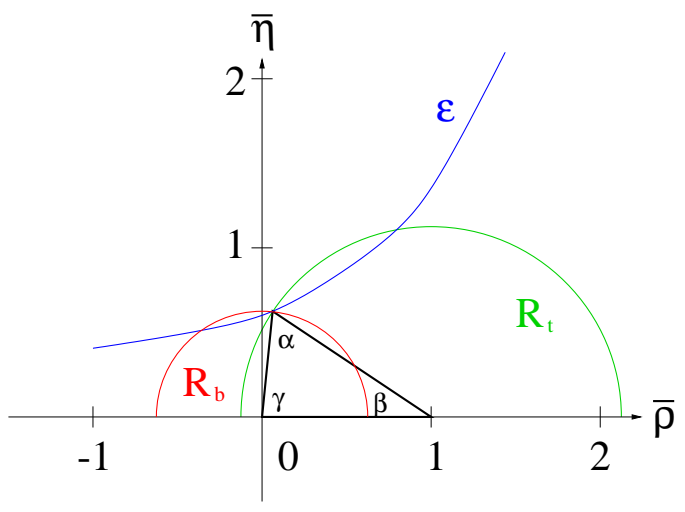

Figure 3. Contours to determine the unitarity triangle in the $\bar{\rho}-\bar{\eta}$ plane.

and has radius $R_{t}$. Finally, using experimental information on an observable $\varepsilon$, which describes "indirect" $C P$ violation in the neutral kaon system and will be discussed in the next section, a hyperbola in the $\bar{\rho}-\bar{\eta}$ plane can be fixed. These contours are sketched in Fig. 3; their intersection gives the apex of the unitarity triangle shown in Fig. 2 (a). The contours that are implied by $B_{d}^{0}-\overline{B_{d}^{0}}$ mixing and $\varepsilon$ depend on $\left|V_{c b}\right|$, the top-quark mass, QCD corrections, and non-perturbative parameters (for a review, see [4]). This feature leads to strong correlations between theoretical and experimental uncertainties. A detailed recent analysis was performed by Ali and London [21], who find the following ranges:

$$
75^{\circ} \leq \alpha \leq 121^{\circ}, \quad 16^{\circ} \leq \beta \leq 34^{\circ}, \quad 38^{\circ} \leq \gamma \leq 81^{\circ} .
$$

We shall come back to this issue in Subsection 8.1, where we emphasize that the present experimental lower bound on $B_{s}^{0}-\overline{B_{s}^{0}}$ mixing has already a very important impact on the allowed range in the $\bar{\rho}-\bar{\eta}$ plane (see Fig. 16).

\section{A Brief Look at CP Violation in the Kaon System}

Although the discovery of $C P$ violation goes back to 1964 [1], so far this phenomenon could only be observed in the $K$-meson system. Here it is described by two complex quantities, called $\varepsilon$ and $\varepsilon^{\prime}$, which are defined by the following ratios of decay amplitudes:

$$
\frac{A\left(K_{\mathrm{L}} \rightarrow \pi^{+} \pi^{-}\right)}{A\left(K_{\mathrm{S}} \rightarrow \pi^{+} \pi^{-}\right)}=\varepsilon+\varepsilon^{\prime}, \quad \frac{A\left(K_{\mathrm{L}} \rightarrow \pi^{0} \pi^{0}\right)}{A\left(K_{\mathrm{S}} \rightarrow \pi^{0} \pi^{0}\right)}=\varepsilon-2 \varepsilon^{\prime} .
$$

While $\varepsilon=(2.280 \pm 0.013) \times e^{i \frac{\pi}{4}} \times 10^{-3}$ parametrizes "indirect" $C P$ violation, originating from the fact that the mass eigenstates of the neutral kaon 
system are not $C P$ eigenstates, the quantity $\operatorname{Re}\left(\varepsilon^{\prime} / \varepsilon\right)$ measures "direct" $C P$ violation in $K \rightarrow \pi \pi$ transitions. The $C P$-violating observable $\varepsilon$ plays an important role to constrain the unitarity triangle $[4,21]$ and implies - using reasonable assumptions about certain hadronic parameters - in particular a positive value of the Wolfenstein parameter $\eta$. In 1999, new measurements of $\operatorname{Re}\left(\varepsilon^{\prime} / \varepsilon\right)$ have demonstrated that this observable is nonzero, thereby excluding "superweak" models of $C P$ violation [22]:

$$
\operatorname{Re}\left(\varepsilon^{\prime} / \varepsilon\right)= \begin{cases}(28 \pm 4.1) \times 10^{-4} & (\mathrm{KTeV} \text { Collaboration [23] }), \\ (14 \pm 4.3) \times 10^{-4} & \text { (NA48 Collaboration [24]). }\end{cases}
$$

Unfortunately, the calculations of $\operatorname{Re}\left(\varepsilon^{\prime} / \varepsilon\right)$ are very involved and suffer at present from large hadronic uncertainties [25]. Consequently, this observable does not allow a powerful test of the $C P$-violating sector of the Standard Model, unless the hadronic matrix elements of the relevant operators can be brought under better control.

In order to test the Standard-Model description of $C P$ violation, the rare decays $K_{\mathrm{L}} \rightarrow \pi^{0} \nu \bar{\nu}$ and $K^{+} \rightarrow \pi^{+} \nu \bar{\nu}$ are more promising, and may allow a determination of $\sin (2 \beta)$ with respectable accuracy [26]. Yet it is clear that the kaon system by itself cannot provide the whole picture of $C P$ violation, and therefore it is essential to study $C P$ violation outside this system. In this respect, $B$-meson decays appear to be most promising. There are of course also other interesting probes to explore $C P$ violation, for example, the neutral $D$-meson system or electric dipole moments, which will, however, not be addressed further in these lectures.

\section{Non-leptonic B Decays}

With respect to testing the Standard-Model description of $C P$ violation, the major role is played by non-leptonic $\bar{B}$ decays, which are mediated by $b \rightarrow q_{1} \overline{q_{2}} d(s)$ quark-level transitions $\left(q_{1}, q_{2} \in\{u, d, c, s\}\right)$.

\subsection{CLASSIFICATION}

There are two kinds of topologies contributing to non-leptonic $B$ decays: tree-diagram-like and "penguin" topologies. The latter consist of gluonic (QCD) and electroweak (EW) penguins. In Figs. 4-6, the corresponding leading-order Feynman diagrams are shown. Depending on the flavour content of their final states, we may classify $b \rightarrow q_{1} \overline{q_{2}} d(s)$ decays as follows:

$-q_{1} \neq q_{2} \in\{u, c\}$ : only tree diagrams contribute.

- $q_{1}=q_{2} \in\{u, c\}$ : tree and penguin diagrams contribute.

$-q_{1}=q_{2} \in\{d, s\}$ : only penguin diagrams contribute. 


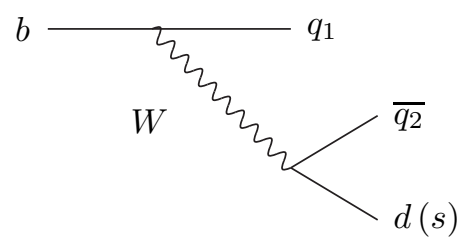

Figure 4. Tree diagrams $\left(q_{1}, q_{2} \in\{u, c\}\right)$.

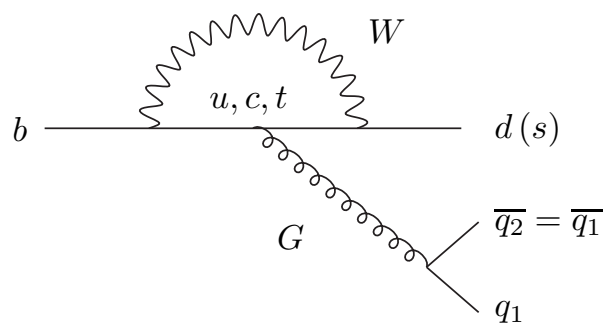

Figure 5. QCD penguin diagrams $\left(q_{1}=q_{2} \in\{u, d, c, s\}\right)$.

\subsection{LOW-ENERGY EFFECTIVE HAMILTONIANS}

In order to analyse non-leptonic $B$ decays theoretically, one uses low-energy effective Hamiltonians, which are calculated by making use of the operator product expansion, yielding transition matrix elements of the following structure:

$$
\left\langle f\left|\mathcal{H}_{\mathrm{eff}}\right| i\right\rangle=\frac{G_{\mathrm{F}}}{\sqrt{2}} \lambda_{\mathrm{CKM}} \sum_{k} C_{k}(\mu)\left\langle f\left|Q_{k}(\mu)\right| i\right\rangle .
$$

The operator product expansion allows us to separate the short-distance contributions to this transition amplitude from the long-distance ones, which are described by perturbative Wilson coefficient functions $C_{k}(\mu)$ and non-perturbative hadronic matrix elements $\left\langle f\left|Q_{k}(\mu)\right| i\right\rangle$, respectively. As usual, $G_{\mathrm{F}}$ is the Fermi constant, $\lambda_{\mathrm{CKM}}$ is a CKM factor, and $\mu$ denotes an appropriate renormalization scale. The $Q_{k}$ are local operators, which are
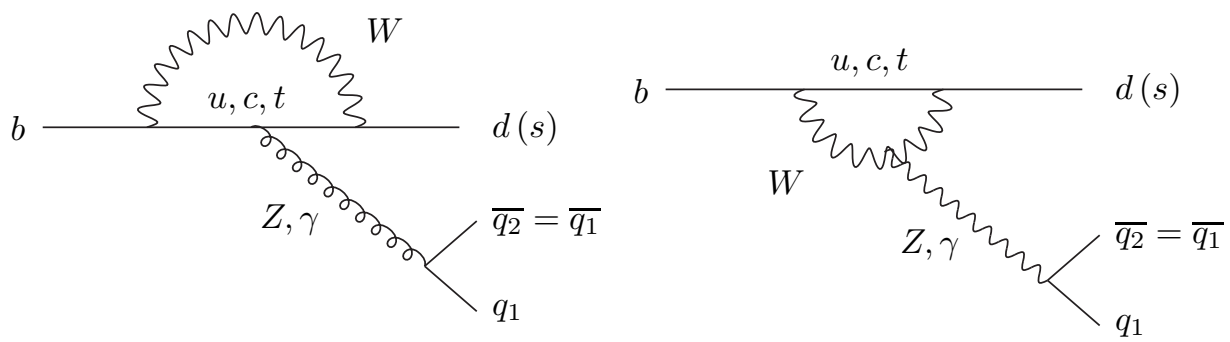

Figure 6. Electroweak penguin diagrams $\left(q_{1}=q_{2} \in\{u, d, c, s\}\right)$. 

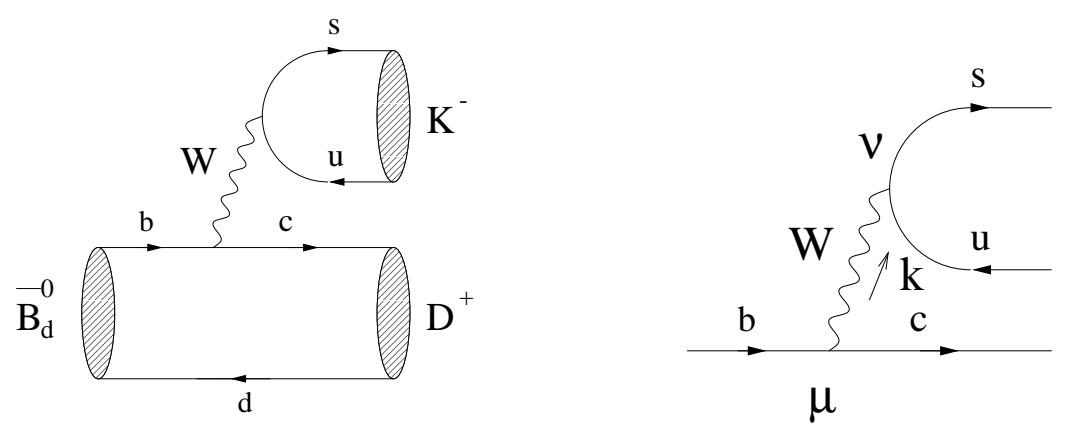

Figure 7. Feynman diagram contributing at leading order to $\overline{B_{d}^{0}} \rightarrow D^{+} K^{-}$.

generated by QCD and electroweak interactions and govern "effectively" the decay in question. The Wilson coefficients $C_{k}(\mu)$ can be considered as scale-dependent couplings related to the vertices described by the $Q_{k}$.

Let us consider $\overline{B_{d}^{0}} \rightarrow D^{+} K^{-}$, a pure "tree" decay, as an example. At leading order, this transition originates from the process shown in Fig. 7. Evaluating the corresponding Feynman diagram yields the amplitude

$$
-\frac{g_{2}^{2}}{8} V_{u s}^{*} V_{c b}\left[\bar{s} \gamma^{\nu}\left(1-\gamma_{5}\right) u\right]\left[\frac{g_{\nu \mu}}{k^{2}-M_{W}^{2}}\right]\left[\bar{c} \gamma^{\mu}\left(1-\gamma_{5}\right) b\right] .
$$

As $k^{2} \approx m_{b}^{2} \ll M_{W}^{2}$, we have

$$
\frac{g_{\nu \mu}}{k^{2}-M_{W}^{2}} \quad \longrightarrow \quad-\frac{g_{\nu \mu}}{M_{W}^{2}} \equiv-\left(\frac{8 G_{\mathrm{F}}}{\sqrt{2} g_{2}^{2}}\right) g_{\nu \mu},
$$

i.e. we may "integrate out" the $W$-boson in (25), and arrive at

$$
\begin{aligned}
\mathcal{H}_{\mathrm{eff}} & =\frac{G_{\mathrm{F}}}{\sqrt{2}} V_{u s}^{*} V_{c b}\left[\bar{s}_{\alpha} \gamma_{\mu}\left(1-\gamma_{5}\right) u_{\alpha}\right]\left[\bar{c}_{\beta} \gamma^{\mu}\left(1-\gamma_{5}\right) b_{\beta}\right] \\
& =\frac{G_{\mathrm{F}}}{\sqrt{2}} V_{u s}^{*} V_{c b}\left(\bar{s}_{\alpha} u_{\alpha}\right)_{\mathrm{V}-\mathrm{A}}\left(\bar{c}_{\beta} b_{\beta}\right)_{\mathrm{V}-\mathrm{A}} \equiv \frac{G_{\mathrm{F}}}{\sqrt{2}} V_{u s}^{*} V_{c b} O_{2},
\end{aligned}
$$

where $\alpha$ and $\beta$ denote $S U(3)_{\mathrm{C}}$ colour indices. Effectively, the vertex shown in Fig. 7 is now described by the "current-current" operator $\mathrm{O}_{2}$ (see Fig. 8).

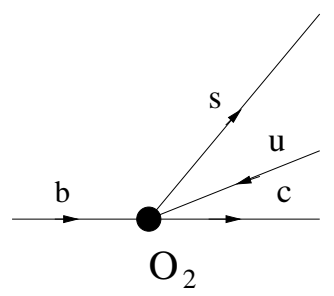

Figure 8. The vertex described by the "current-current" operator $\mathrm{O}_{2}$. 
If we take into account QCD corrections, operator mixing leads to a second "current-current" operator:

$$
O_{1} \equiv\left[\bar{s}_{\alpha} \gamma_{\mu}\left(1-\gamma_{5}\right) u_{\beta}\right]\left[\bar{c}_{\beta} \gamma^{\mu}\left(1-\gamma_{5}\right) b_{\alpha}\right]
$$

Consequently, we obtain a low-energy effective Hamiltonian of the following structure:

$$
\mathcal{H}_{\mathrm{eff}}=\frac{G_{\mathrm{F}}}{\sqrt{2}} V_{u s}^{*} V_{c b}\left[C_{1}(\mu) O_{1}+C_{2}(\mu) O_{2}\right],
$$

where $C_{1}(\mu) \neq 0$ and $C_{2}(\mu) \neq 1$ are due to QCD renormalization effects. In order to evaluate these coefficients, we first have to calculate QCD corrections to the vertices shown in Figs. 7 and 8, and then have to express the QCD-corrected transition amplitude in terms of QCD-corrected matrix elements and Wilson coefficients as in (24). This procedure is called "matching". The results for the $C_{k}(\mu)$ obtained this way contain terms of $\log \left(\mu / M_{W}\right)$, which become large for $\mu=\mathcal{O}\left(m_{b}\right)$, the scale governing the hadronic matrix elements of the $O_{k}$. However, the renormalization group, exploiting the fact that the transition amplitude (24) cannot depend on the

chosen renormalization scale $\mu$, allows us to sum up the following terms of the Wilson coefficients:

$$
\alpha_{s}^{n}\left[\log \left(\frac{\mu}{M_{W}}\right)\right]^{n}(\mathrm{LO}), \quad \alpha_{s}^{n}\left[\log \left(\frac{\mu}{M_{W}}\right)\right]^{n-1}(\mathrm{NLO}), \quad \ldots
$$

A detailed discussion of these techniques can be found in Refs. [27, 28].

\subsection{DECAYS WITH TREE AND PENGUIN CONTRIBUTIONS}

In order to explore $C P$ violation in the $B$ system, non-leptonic decays receiving both tree and penguin contributions, i.e. $|\Delta B|=1, \Delta C=\Delta U=0$ modes (see Subsection 4.1), play an outstanding role. In this case, because of the penguin topologies, the operator basis is much larger than in our example (29), where we considered a pure "tree" decay. We obtain

$$
\mathcal{H}_{\mathrm{eff}}=\frac{G_{\mathrm{F}}}{\sqrt{2}}\left[\sum_{j=u, c} V_{j r}^{*} V_{j b}\left\{\sum_{k=1}^{2} C_{k}(\mu) Q_{k}^{j r}+\sum_{k=3}^{10} C_{k}(\mu) Q_{k}^{r}\right\}\right],
$$

where the operators $Q_{k}^{j r}(j \in\{u, c\}, r \in\{d, s\})$ can be divided as follows:

- Current-current operators:

$$
\begin{aligned}
Q_{1}^{j r} & =\left(\bar{r}_{\alpha} j_{\beta}\right)_{\mathrm{V}-\mathrm{A}}\left(\bar{j}_{\beta} b_{\alpha}\right)_{\mathrm{V}-\mathrm{A}} \\
Q_{2}^{j r} & =\left(\bar{r}_{\alpha} j_{\alpha}\right)_{\mathrm{V}-\mathrm{A}}\left(\bar{j}_{\beta} b_{\beta}\right)_{\mathrm{V}-\mathrm{A}} .
\end{aligned}
$$


- QCD penguin operators:

$$
\begin{aligned}
Q_{3}^{r} & =\left(\bar{r}_{\alpha} b_{\alpha}\right)_{\mathrm{V}-\mathrm{A}} \sum_{q^{\prime}}\left(\bar{q}_{\beta}^{\prime} q_{\beta}^{\prime}\right)_{\mathrm{V}-\mathrm{A}} \\
Q_{4}^{r} & =\left(\bar{r}_{\alpha} b_{\beta}\right)_{\mathrm{V}-\mathrm{A}} \sum_{q^{\prime}}\left(\bar{q}_{\beta}^{\prime} q_{\alpha}^{\prime}\right)_{\mathrm{V}-\mathrm{A}} \\
Q_{5}^{r} & =\left(\bar{r}_{\alpha} b_{\alpha}\right)_{\mathrm{V}-\mathrm{A}} \sum_{q^{\prime}}\left(\bar{q}_{\beta}^{\prime} q_{\beta}^{\prime}\right)_{\mathrm{V}+\mathrm{A}} \\
Q_{6}^{r} & =\left(\bar{r}_{\alpha} b_{\beta}\right)_{\mathrm{V}-\mathrm{A}} \sum_{q^{\prime}}\left(\bar{q}_{\beta}^{\prime} q_{\alpha}^{\prime}\right)_{\mathrm{V}+\mathrm{A}} .
\end{aligned}
$$

- Electroweak (EW) penguin operators (the $e_{q^{\prime}}$ denote quark charges):

$$
\begin{aligned}
& Q_{7}^{r}=\frac{3}{2}\left(\bar{r}_{\alpha} b_{\alpha}\right)_{\mathrm{V}-\mathrm{A}} \sum_{q^{\prime}} e_{q^{\prime}}\left(\bar{q}_{\beta}^{\prime} q_{\beta}^{\prime}\right)_{\mathrm{V}+\mathrm{A}} \\
& Q_{8}^{r}=\frac{3}{2}\left(\bar{r}_{\alpha} b_{\beta}\right)_{\mathrm{V}-\mathrm{A}} \sum_{q^{\prime}} e_{q^{\prime}}\left(\bar{q}_{\beta}^{\prime} q_{\alpha}^{\prime}\right)_{\mathrm{V}+\mathrm{A}} \\
& Q_{9}^{r}=\frac{3}{2}\left(\bar{r}_{\alpha} b_{\alpha}\right)_{\mathrm{V}-\mathrm{A}} \sum_{q^{\prime}} e_{q^{\prime}}\left(\bar{q}_{\beta}^{\prime} q_{\beta}^{\prime}\right)_{\mathrm{V}-\mathrm{A}} \\
& Q_{10}^{r}=\frac{3}{2}\left(\bar{r}_{\alpha} b_{\beta}\right)_{\mathrm{V}-\mathrm{A}} \sum_{q^{\prime}} e_{q^{\prime}}\left(\bar{q}_{\beta}^{\prime} q_{\alpha}^{\prime}\right)_{\mathrm{V}-\mathrm{A}} \text {. }
\end{aligned}
$$

The current-current, QCD and EW penguin operators are related to the tree, QCD and EW penguin processes shown in Figs. 4-6. At a renormalization scale $\mu=\mathcal{O}\left(m_{b}\right)$, the Wilson coefficients of the current-current operators are $C_{1}(\mu)=\mathcal{O}\left(10^{-1}\right)$ and $C_{2}(\mu)=\mathcal{O}(1)$, whereas those of the penguin operators are $\mathcal{O}\left(10^{-2}\right)$. The calculation of (31) beyond the leading logarithmic approximation (LO) has been reviewed in [28], where also numerical values of the corresponding (NLO) Wilson coefficients are given.

\subsection{ELECTROWEAK PENGUIN EFFECTS}

Since the ratio $\alpha / \alpha_{s}=\mathcal{O}\left(10^{-2}\right)$ of the QED and QCD couplings is very small, we expect naively that EW penguins should play a minor role in comparison with QCD penguins. This would actually be the case if the top quark was not "heavy". However, since the Wilson coefficient of the EW penguin operator $Q_{9}$ increases strongly with the top-quark mass $m_{t}$, we obtain interesting EW penguin effects in several $B$ decays: $B^{-} \rightarrow K^{-} \phi$ is affected significantly by EW penguins, whereas $B \rightarrow \pi \phi$ and $B_{s} \rightarrow \pi^{0} \phi$ are even dominated by such topologies [29, 30]. Electroweak penguins have also an important impact on $B \rightarrow \pi K$ modes [31], as we will discuss in more detail in Section 9.

\subsection{FACTORIZATION OF HADRONIC MATRIX ELEMENTS}

In order to discuss "factorization", let us consider again our example from Subsection 4.2 , the decay $\overline{B_{d}^{0}} \rightarrow D^{+} K^{-}$. The problem in the evaluation of the corresponding transition amplitude is the calculation of the hadronic matrix elements of the $O_{1,2}$ operators between the $\left\langle K^{-} D^{+}\right|$final and $\left|\overline{B_{d}^{0}}\right\rangle$ initial states. Making use of the well-known $S U\left(N_{\mathrm{C}}\right)$ colour-algebra relation

$$
T_{\alpha \beta}^{a} T_{\gamma \delta}^{a}=\frac{1}{2}\left(\delta_{\alpha \delta} \delta_{\beta \gamma}-\frac{1}{N_{\mathrm{C}}} \delta_{\alpha \beta} \delta_{\gamma \delta}\right)
$$


to re-write the operator $O_{1}$, we obtain

$$
\begin{gathered}
\left\langle K^{-} D^{+}\left|\mathcal{H}_{\mathrm{eff}}\right| \overline{B_{d}^{0}}\right\rangle=\frac{G_{\mathrm{F}}}{\sqrt{2}} V_{u s}^{*} V_{c b}\left[a_{1}\left\langle K^{-} D^{+}\left|\left(\bar{s}_{\alpha} u_{\alpha}\right)_{\mathrm{V}-\mathrm{A}}\left(\bar{c}_{\beta} b_{\beta}\right)_{\mathrm{V}-\mathrm{A}}\right| \overline{B_{d}^{0}}\right\rangle\right. \\
\left.+2 C_{1}\left\langle K^{-} D^{+}\left|\left(\bar{s}_{\alpha} T_{\alpha \beta}^{a} u_{\beta}\right)_{\mathrm{V}-\mathrm{A}}\left(\bar{c}_{\gamma} T_{\gamma \delta}^{a} b_{\delta}\right)_{\mathrm{V}-\mathrm{A}}\right| \overline{B_{d}^{0}}\right\rangle\right]
\end{gathered}
$$

with

$$
a_{1}=\frac{C_{1}}{N_{\mathrm{C}}}+C_{2} .
$$

It is now straightforward to "factorize" the hadronic matrix elements:

$$
\begin{aligned}
\left\langle K^{-}\right. & \left.D^{+}\left|\left(\bar{s}_{\alpha} u_{\alpha}\right)_{\mathrm{V}-\mathrm{A}}\left(\bar{c}_{\beta} b_{\beta}\right)_{\mathrm{V}-\mathrm{A}}\right| \overline{B_{d}^{0}}\right\rangle\left.\right|_{\text {fact }} \\
& =\left\langle K^{-}\left|\left[\bar{s}_{\alpha} \gamma_{\mu}\left(1-\gamma_{5}\right) u_{\alpha}\right]\right| 0\right\rangle\left\langle D^{+}\left|\left[\bar{c}_{\beta} \gamma^{\mu}\left(1-\gamma_{5}\right) b_{\beta}\right]\right| \overline{B_{d}^{0}}\right\rangle \\
& \propto f_{K}\left(\text { "decay constant") } \times F_{B D} \text { ("form factor") },\right. \\
& \left.\left\langle K^{-} D^{+}\left|\left(\bar{s}_{\alpha} T_{\alpha \beta}^{a} u_{\beta}\right)_{\mathrm{V}-\mathrm{A}}\left(\bar{c}_{\gamma} T_{\gamma \delta}^{a} b_{\delta}\right)_{\mathrm{V}-\mathrm{A}}\right| \overline{B_{d}^{0}}\right\rangle\right|_{\text {fact }}=0 .
\end{aligned}
$$

The quantity introduced in (37) is a phenomenological "colour factor", governing "colour-allowed" decays. In the case of "colour-suppressed" modes, for instance $\overline{B_{d}^{0}} \rightarrow \pi^{0} D^{0}$, we have to deal with the combination

$$
a_{2}=C_{1}+\frac{C_{2}}{N_{\mathrm{C}}} .
$$

The concept of "factorization" of hadronic matrix elements has a long history [32], and can be justified, for example, in the large $N_{\mathrm{C}}$ limit [33]. Recently, an interesting approach was proposed in Ref. [34], which may provide an important step towards a rigorous basis for factorization for a large class of non-leptonic two-body $B$-meson decays in the heavy-quark limit. The resulting "factorization" formula incorporates elements both of the "naive" factorization approach sketched above and of the hard-scattering picture. Let us consider a decay $\bar{B} \rightarrow M_{1} M_{2}$, where $M_{1}$ picks up the spectator quark. If $M_{1}$ is either a heavy $(D)$ or a light $(\pi, K)$ meson, and $M_{2}$ a light $(\pi, K)$ meson, a "QCD factorization" formula of the following structure can be derived: ${ }^{2}$

$$
\begin{aligned}
& A\left(\bar{B} \rightarrow M_{1} M_{2}\right)=[\text { "naive factorization"] } \\
& \quad \times\left[1+\text { calculable } \mathcal{O}\left(\alpha_{s}\right)+\mathcal{O}\left(\Lambda_{\mathrm{QCD}} / m_{b}\right)\right] .
\end{aligned}
$$

Whereas the $\mathcal{O}\left(\alpha_{s}\right)$ terms, i.e. the radiative non-factorizable corrections to "naive" factorization, can be calculated in a systematic way, the main limitation is due to the $\mathcal{O}\left(\Lambda_{\mathrm{QCD}} / m_{b}\right)$ terms, which require further studies.

2 "QCD factorization" does not hold, if $M_{2}$ is a heavy $(D)$ meson. 

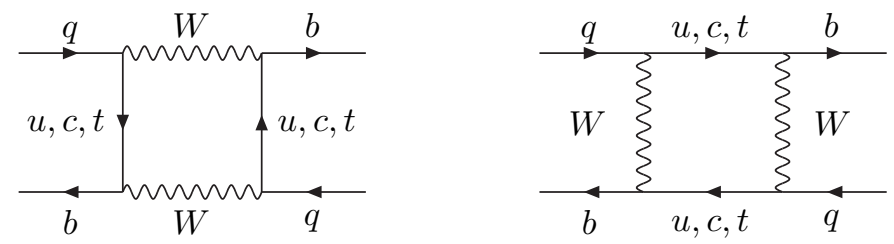

Figure 9. Box diagrams contributing to $B_{q}^{0}-\overline{B_{q}^{0}}$ mixing $(q \in\{d, s\})$.

\section{The Formalism of $B_{d, s}^{0}-\overline{B_{d, s}^{0}}$ Mixing}

Within the Standard Model, $B_{q}^{0}-\overline{B_{q}^{0}}$ mixing $(q \in\{d, s\})$ is induced at lowest order through the box diagrams shown in Fig. 9. The Wigner-Weisskopf formalism yields an effective Schrödinger equation

$$
i \frac{\partial}{\partial t}\left(\begin{array}{c}
a(t) \\
b(t)
\end{array}\right)=\left[\left(\begin{array}{cc}
M_{0}^{(q)} & M_{12}^{(q)} \\
M_{12}^{(q) *} & M_{0}^{(q)}
\end{array}\right)-\frac{i}{2}\left(\begin{array}{cc}
\Gamma_{0}^{(q)} & \Gamma_{12}^{(q)} \\
\Gamma_{12}^{(q) *} & \Gamma_{0}^{(q)}
\end{array}\right)\right] \cdot\left(\begin{array}{c}
a(t) \\
b(t)
\end{array}\right)
$$

which describes the time evolution of the state vector

$$
\left|\psi_{q}(t)\right\rangle=a(t)\left|B_{q}^{0}\right\rangle+b(t)\left|\overline{B_{q}^{0}}\right\rangle .
$$

\subsection{SOLUTION OF THE SCHRÖDINGER EQUATION}

It is straightforward to calculate the eigenstates $\left|B_{ \pm}^{(q)}\right\rangle$ and eigenvalues $\lambda_{ \pm}^{(q)}$ of the Hamiltonian given in (42):

$$
\begin{gathered}
\left|B_{ \pm}^{(q)}\right\rangle=\frac{1}{\sqrt{1+\left|\alpha_{q}\right|^{2}}}\left(\left|B_{q}^{0}\right\rangle \pm \alpha_{q}\left|\overline{B_{q}^{0}}\right\rangle\right) \\
\lambda_{ \pm}^{(q)}=\left(M_{0}^{(q)}-\frac{i}{2} \Gamma_{0}^{(q)}\right) \pm\left(M_{12}^{(q)}-\frac{i}{2} \Gamma_{12}^{(q)}\right) \alpha_{q}
\end{gathered}
$$

where

$$
\alpha_{q} e^{+i\left(\Theta_{\Gamma_{12}}^{(q)}+n^{\prime} \pi\right)}=\sqrt{\frac{4\left|M_{12}^{(q)}\right|^{2} e^{-i 2 \delta \Theta_{M / \Gamma}^{(q)}}+\left|\Gamma_{12}^{(q)}\right|^{2}}{4\left|M_{12}^{(q)}\right|^{2}+\left|\Gamma_{12}^{(q)}\right|^{2}-4\left|M_{12}^{(q)}\right|\left|\Gamma_{12}^{(q)}\right| \sin \delta \Theta_{M / \Gamma}^{(q)}} .}
$$

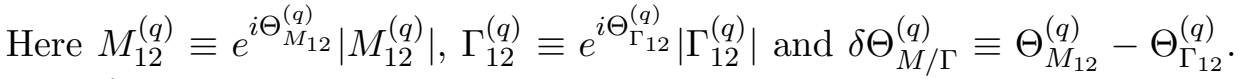
The $n^{\prime} \in\{0,1\}$ parametrizes the sign of the square root appearing in that 
expression. Calculating the dispersive parts of the box diagrams gives [4]

$$
M_{12}^{(q)}=\frac{G_{\mathrm{F}}^{2} M_{W}^{2} \eta_{B} m_{B_{q}} B_{B_{q}} F_{B_{q}}^{2}}{12 \pi^{2}}\left(V_{t q}^{*} V_{t b}\right)^{2} S_{0}\left(x_{t}\right) e^{i\left(\pi-\phi_{\mathrm{CP}}\left(B_{q}\right)\right)},
$$

where $\eta_{B}$ is a perturbative QCD correction, $m_{B_{q}}$ the $B_{q}$-meson mass, $B_{B_{q}}$ a non-perturbative "bag" parameter related to $\left\langle\overline{B_{q}^{0}}\left|\left[\bar{b} \gamma_{\mu}\left(1-\gamma_{5}\right) q\right]^{2}\right| B_{q}^{0}\right\rangle, F_{B_{q}}$ the $B_{q}$ decay constant, $x_{t} \equiv m_{t}^{2} / M_{W}^{2}, S_{0}\left(x_{t}\right)=\mathcal{O}(1)$, and

$$
(\mathcal{C P})\left|B_{q}^{0}\right\rangle=e^{i \phi_{\mathrm{CP}}\left(B_{q}\right)}\left|\overline{B_{q}^{0}}\right\rangle \text {. }
$$

Moreover, we obtain from the absorptive parts of the boxes:

$$
\frac{\Gamma_{12}^{(q)}}{M_{12}^{(q)}} \approx-\frac{3 \pi}{2 S_{0}\left(x_{t}\right)} \frac{m_{b}^{2}}{M_{W}^{2}}=\mathcal{O}\left(m_{b}^{2} / m_{t}^{2}\right) \ll 1 .
$$

Consequently, neglecting 2nd order terms, we arrive at

$$
\alpha_{q}=\left[1+\frac{\left|\Gamma_{12}^{(q)}\right|}{2\left|M_{12}^{(q)}\right|} \sin \delta \Theta_{M / \Gamma}^{(q)}\right] e^{-i\left(\Theta_{M_{12}}^{(q)}+n^{\prime} \pi\right)} .
$$

The deviation of $\left|\alpha_{q}\right|$ from 1 describes $C P$ violation in $B_{q}^{0}-\overline{B_{q}^{0}}$ oscillations, and can be probed through "wrong-charge" lepton asymmetries:

$$
\begin{gathered}
\mathcal{A}_{\mathrm{SL}}^{(q)} \equiv \frac{\Gamma\left(B_{q}^{0}(t) \rightarrow l^{-} \bar{\nu}_{l} X\right)-\Gamma\left(\overline{B_{q}^{0}}(t) \rightarrow l^{+} \nu_{l} X\right)}{\Gamma\left(B_{q}^{0}(t) \rightarrow l^{-} \bar{\nu}_{l} X\right)+\Gamma\left(\overline{B_{q}^{0}}(t) \rightarrow l^{+} \nu_{l} X\right)} \\
=\frac{\left|\alpha_{q}\right|^{4}-1}{\left|\alpha_{q}\right|^{4}+1} \approx \frac{\left|\Gamma_{12}^{(q)}\right|}{\left|M_{12}^{(q)}\right|} \sin \delta \Theta_{M / \Gamma}^{(q)} .
\end{gathered}
$$

Note that the time dependences cancel in (51). Because of

$$
\left|\Gamma_{12}^{(q)}\right| /\left|M_{12}^{(q)}\right| \propto m_{b}^{2} / m_{t}^{2}, \quad \sin \delta \Theta_{M / \Gamma}^{(q)} \propto m_{c}^{2} / m_{b}^{2},
$$

the asymmetry $\mathcal{A}_{\mathrm{SL}}^{(q)}$ is suppressed by a factor $m_{c}^{2} / m_{t}^{2}=\mathcal{O}\left(10^{-4}\right)$, and is hence very small in the Standard Model. Consequently, it represents an interesting probe to search for new physics.

\subsection{TIME EVOLUTION}

The time evolution of initially, i.e. at $t=0$, pure $\left|B_{q}^{0}\right\rangle$ - and $\left|\overline{B_{q}^{0}}\right\rangle$-meson states is given by

$$
\begin{aligned}
& \left|B_{q}^{0}(t)\right\rangle=f_{+}^{(q)}(t)\left|B_{q}^{0}\right\rangle+\alpha_{q} f_{-}^{(q)}(t)\left|\overline{B_{q}^{0}}\right\rangle \\
& \left|\overline{B_{q}^{0}}(t)\right\rangle=\frac{1}{\alpha_{q}} f_{-}^{(q)}(t)\left|B_{q}^{0}\right\rangle+f_{+}^{(q)}(t)\left|\overline{B_{q}^{0}}\right\rangle
\end{aligned}
$$


where

$$
f_{ \pm}^{(q)}(t)=\frac{1}{2}\left[e^{-i \lambda_{+}^{(q)} t} \pm e^{-i \lambda_{-}^{(q)} t}\right] .
$$

These time-dependent state vectors allow the calculation of the corresponding transition rates. To this end, it is useful to introduce

$$
\begin{aligned}
\left|g_{ \pm}^{(q)}(t)\right|^{2} & =\frac{1}{4}\left[e^{-\Gamma_{\mathrm{L}}^{(q)} t}+e^{-\Gamma_{\mathrm{H}}^{(q)} t} \pm 2 e^{-\Gamma_{q} t} \cos \left(\Delta M_{q} t\right)\right] \\
g_{-}^{(q)}(t) g_{+}^{(q)}(t)^{*} & =\frac{1}{4}\left[e^{-\Gamma_{\mathrm{L}}^{(q)} t}-e^{-\Gamma_{\mathrm{H}}^{(q)} t}+2 i e^{-\Gamma_{q} t} \sin \left(\Delta M_{q} t\right)\right],
\end{aligned}
$$

and

$$
\xi_{f}^{(q)}=e^{-i \Theta_{M_{12}}^{(q)}} \frac{A\left(\overline{B_{q}^{0}} \rightarrow f\right)}{A\left(B_{q}^{0} \rightarrow f\right)}, \quad \xi_{\bar{f}}^{(q)}=e^{-i \Theta_{M_{12}}^{(q)}} \frac{A\left(\overline{B_{q}^{0}} \rightarrow \bar{f}\right)}{A\left(B_{q}^{0} \rightarrow \bar{f}\right)}
$$

where

$$
\Theta_{M_{12}}^{(q)}=\pi+2 \arg \left(V_{t q}^{*} V_{t b}\right)-\phi_{\mathrm{CP}}\left(B_{q}\right)
$$

is the $C P$-violating weak $B_{q}^{0}-\overline{B_{q}^{0}}$ mixing phase. Whereas $\Theta_{M_{12}}^{(q)}$ depends on the chosen CKM and $C P$ phase conventions, $\xi_{f}^{(q)}$ and $\xi_{\bar{f}}^{(q)}$ are conventionindependent observables.

The $g_{ \pm}^{(q)}(t)$ are related to the $f_{ \pm}^{(q)}(t)$. However, whereas the latter functions depend on $n^{\prime}$, the $g_{ \pm}^{(q)}(t)$ do not depend on this parameter. The $n^{\prime}$ dependence is cancelled by introducing the positive mass difference

$$
\Delta M_{q} \equiv M_{\mathrm{H}}^{(q)}-M_{\mathrm{L}}^{(q)}=2\left|M_{12}^{(q)}\right|>0
$$

of the mass eigenstates $\left|B_{q}^{\mathrm{H}}\right\rangle$ ("heavy") and $\left|B_{q}^{\mathrm{L}}\right\rangle$ ("light"). The quantities $\Gamma_{\mathrm{H}}^{(q)}$ and $\Gamma_{\mathrm{L}}^{(q)}$ denote the corresponding decay widths. Their difference can be expressed as

$$
\Delta \Gamma_{q} \equiv \Gamma_{\mathrm{H}}^{(q)}-\Gamma_{\mathrm{L}}^{(q)}=\frac{4 \operatorname{Re}\left[M_{12}^{(q)} \Gamma_{12}^{(q) *}\right]}{\Delta M_{q}},
$$

whereas their average is given by

$$
\Gamma_{q} \equiv \frac{\Gamma_{\mathrm{H}}^{(q)}+\Gamma_{\mathrm{L}}^{(q)}}{2}=\Gamma_{0}^{(q)} .
$$

There is the following interesting relation:

$$
\frac{\Delta \Gamma_{q}}{\Gamma_{q}} \approx-\frac{3 \pi}{2 S_{0}\left(x_{t}\right)} \frac{m_{b}^{2}}{M_{W}^{2}} x_{q}=\mathcal{O}\left(10^{-2}\right) \times x_{q},
$$


where

$$
x_{q} \equiv \frac{\Delta M_{q}}{\Gamma_{q}}=\left\{\begin{array}{cc}
0.723 \pm 0.032 & (q=d) \\
\mathcal{O}(20) & (q=s)
\end{array}\right.
$$

denotes the $B_{q}^{0}-\overline{B_{q}^{0}}$ "mixing parameter". Consequently, there may be a sizeable width difference in the $B_{s}$ system, whereas $\Delta \Gamma_{d}$ is expected to be negligibly small. We shall come back to $\Delta \Gamma_{s}$ in Section 8 .

Combining the formulae listed above, we arrive at the following transition rates for decays of initially, i.e. at $t=0$, present $B_{q}^{0}$ - and $\overline{B_{q}^{0}}$-mesons:

$$
\begin{aligned}
& \Gamma\left(\stackrel{-\left(B_{q}^{0}\right.}{0}(t) \rightarrow f\right) \\
& \quad=\left[\left|g_{\mp}^{(q)}(t)\right|^{2}+\left|\xi_{f}^{(q)}\right|^{2}\left|g_{ \pm}^{(q)}(t)\right|^{2}-2 \operatorname{Re}\left\{\xi_{f}^{(q)} g_{ \pm}^{(q)}(t) g_{\mp}^{(q)}(t)^{*}\right\}\right] \tilde{\Gamma}_{f},(
\end{aligned}
$$

where the time-independent rate $\tilde{\Gamma}_{f}$ corresponds to the "unevolved" decay amplitude $A\left(B_{q}^{0} \rightarrow f\right)$, which can be calculated by performing the usual phase-space integrations. The rates into the $C P$-conjugate final state $\bar{f}$ can be obtained straightforwardly from (65) through the substitutions

$$
\tilde{\Gamma}_{f} \rightarrow \tilde{\Gamma}_{\bar{f}}, \quad \xi_{f}^{(q)} \rightarrow \xi_{\bar{f}}^{(q)} .
$$

\subsection{CP-VIOLATING ASYMMETRIES}

A particularly simple and interesting situation arises if we restrict ourselves to decays of neutral $B_{q}$-mesons into $C P$ self-conjugate final states $|f\rangle$, satisfying the relation

$$
(\mathcal{C P})|f\rangle= \pm|f\rangle
$$

Consequently, we have $\xi_{f}^{(q)}=\xi_{\frac{f}{f}}^{(q)}$ in this case (see (58)). Using (65), the corresponding time-dependent $C P$ asymmetry can be expressed as

$$
\begin{aligned}
& a_{\mathrm{CP}}(t) \equiv \frac{\Gamma\left(B_{q}^{0}(t) \rightarrow f\right)-\Gamma\left(\overline{B_{q}^{0}}(t) \rightarrow f\right)}{\Gamma\left(B_{q}^{0}(t) \rightarrow f\right)+\Gamma\left(\overline{B_{q}^{0}}(t) \rightarrow f\right)} \\
&=\left[\frac{\mathcal{A}_{\mathrm{CP}}^{\mathrm{dir}}\left(B_{q} \rightarrow f\right) \cos \left(\Delta M_{q} t\right)+\mathcal{A}_{\mathrm{CP}}^{\operatorname{mix}}\left(B_{q} \rightarrow f\right) \sin \left(\Delta M_{q} t\right)}{\cosh \left(\Delta \Gamma_{q} t / 2\right)-\mathcal{A}_{\Delta \Gamma}\left(B_{q} \rightarrow f\right) \sinh \left(\Delta \Gamma_{q} t / 2\right)}\right],
\end{aligned}
$$

where we have separated the "direct" from the "mixing-induced" $C P$ violating contributions, which are described by

$$
\mathcal{A}_{\mathrm{CP}}^{\mathrm{dir}}\left(B_{q} \rightarrow f\right) \equiv \frac{1-\left|\xi_{f}^{(q)}\right|^{2}}{1+\left|\xi_{f}^{(q)}\right|^{2}} \quad \text { and } \quad \mathcal{A}_{\mathrm{CP}}^{\mathrm{mix}}\left(B_{q} \rightarrow f\right) \equiv \frac{2 \operatorname{Im} \xi_{f}^{(q)}}{1+\left|\xi_{f}^{(q)}\right|^{2}},
$$


respectively. The terminology "direct $C P$ violation" refers to $C P$-violating effects, which arise directly in the corresponding decay amplitudes and are due to interference between different CKM amplitudes. On the other hand, "mixing-induced $C P$ violation" originates from interference effects between $B_{q}^{0}-\overline{B_{q}^{0}}$ mixing and decay processes. The width difference $\Delta \Gamma_{q}$, which may be sizeable in the $B_{s}$ system, provides another observable

$$
\mathcal{A}_{\Delta \Gamma}\left(B_{q} \rightarrow f\right) \equiv \frac{2 \operatorname{Re} \xi_{f}^{(q)}}{1+\left|\xi_{f}^{(q)}\right|^{2}},
$$

which is, however, not independent from $\mathcal{A}_{\mathrm{CP}}^{\mathrm{dir}}\left(B_{q} \rightarrow f\right)$ and $\mathcal{A}_{\mathrm{CP}}^{\operatorname{mix}}\left(B_{q} \rightarrow f\right)$ :

$$
\left[\mathcal{A}_{\mathrm{CP}}^{\mathrm{dir}}\left(B_{q} \rightarrow f\right)\right]^{2}+\left[\mathcal{A}_{\mathrm{CP}}^{\mathrm{mix}}\left(B_{q} \rightarrow f\right)\right]^{2}+\left[\mathcal{A}_{\Delta \Gamma}\left(B_{q} \rightarrow f\right)\right]^{2}=1 .
$$

In order to calculate the observable $\xi_{f}^{(q)}$, containing essentially all the information needed to evaluate the $C P$ asymmetry (68), we employ the low-energy effective Hamiltonian (31):

$$
\begin{aligned}
& A\left(\overline{B_{q}^{0}} \rightarrow f\right)=\left\langle f\left|\mathcal{H}_{\mathrm{eff}}(\Delta B=-1)\right| \overline{B_{q}^{0}}\right\rangle= \\
& \quad\left\langle f\left|\frac{G_{\mathrm{F}}}{\sqrt{2}}\left[\sum_{j=u, c} V_{j r}^{*} V_{j b}\left\{\sum_{k=1}^{2} C_{k}(\mu) Q_{k}^{j r}(\mu)+\sum_{k=3}^{10} C_{k}(\mu) Q_{k}^{r}(\mu)\right\}\right]\right| \overline{B_{q}^{0}}\right\rangle,
\end{aligned}
$$

where $r \in\{d, s\}$ distinguishes between $b \rightarrow d$ and $b \rightarrow s$ transitions. On the other hand, we also have

$$
\begin{aligned}
& A\left(B_{q}^{0} \rightarrow f\right)=\left\langle f\left|\mathcal{H}_{\mathrm{eff}}(\Delta B=-1)^{\dagger}\right| B_{q}^{0}\right\rangle= \\
& \left\langle f\left|\frac{G_{\mathrm{F}}}{\sqrt{2}}\left[\sum_{j=u, c} V_{j r} V_{j b}^{*}\left\{\sum_{k=1}^{2} C_{k}(\mu) Q_{k}^{j r \dagger}(\mu)+\sum_{k=3}^{10} C_{k}(\mu) Q_{k}^{r \dagger}(\mu)\right\}\right]\right| B_{q}^{0}\right\rangle .
\end{aligned}
$$

Performing appropriate $C P$ transformations in this expression, i.e. inserting the operator $(\mathcal{C P})^{\dagger}(\mathcal{C P})=\hat{1}$ both after $\langle f|$ and in front of $\left|B_{q}^{0}\right\rangle$, yields

$$
\begin{aligned}
& A\left(B_{q}^{0} \rightarrow f\right)= \pm e^{i \phi_{\mathrm{CP}}\left(B_{q}\right)} \times \\
& \quad\langle f| \frac{G_{\mathrm{F}}}{\sqrt{2}}\left[\sum_{j=u, c} V_{j r} V_{j b}^{*}\left\{\sum_{k=1}^{2} C_{k}(\mu) Q_{k}^{j r}(\mu)+\sum_{k=3}^{10} C_{k}(\mu) Q_{k}^{r}(\mu)\right\}|| \overline{B_{q}^{0}}\right\rangle
\end{aligned}
$$

where we have applied the relation $(\mathcal{C P}) Q_{k}^{j r \dagger}(\mathcal{C P})^{\dagger}=Q_{k}^{j r}$, and have furthermore taken into account (48). Using now (58) and (59), we finally arrive 
at

$$
\xi_{f}^{(q)}=\mp e^{-i \phi_{q}}\left[\frac{\sum_{j=u, c} V_{j r}^{*} V_{j b}\left\langle f\left|\mathcal{Q}^{j r}\right| \overline{B_{q}^{0}}\right\rangle}{\sum_{j=u, c} V_{j r} V_{j b}^{*}\left\langle f\left|\mathcal{Q}^{j r}\right| \overline{B_{q}^{0}}\right\rangle}\right],
$$

where

$$
\mathcal{Q}^{j r} \equiv \sum_{k=1}^{2} C_{k}(\mu) Q_{k}^{j r}+\sum_{k=3}^{10} C_{k}(\mu) Q_{k}^{r},
$$

and where

$$
\phi_{q} \equiv 2 \arg \left(V_{t q}^{*} V_{t b}\right)=\left\{\begin{array}{cc}
+2 \beta & (q=d) \\
-2 \delta \gamma & (q=s)
\end{array}\right.
$$

is related to the weak $B_{q}^{0}-\overline{B_{q}^{0}}$ mixing phase. Note that the phase-conventiondependent quantity $\phi_{\mathrm{CP}}\left(B_{q}\right)$ cancels in this expression.

In general, the observable $\xi_{f}^{(q)}$ suffers from large hadronic uncertainties, which are introduced by the hadronic matrix elements in Eq. (75). However, if the decay $B_{q} \rightarrow f$ is dominated by a single CKM amplitude, i.e.

$$
A\left(B_{q}^{0} \rightarrow f\right)=e^{-i \phi_{f} / 2}\left[e^{i \delta_{f}}\left|M_{f}\right|\right],
$$

the strong matrix element $e^{i \delta_{f}}\left|M_{f}\right|$ cancels, and $\xi_{f}^{(q)}$ takes the simple form

$$
\xi_{f}^{(q)}=\mp \exp \left[-i\left(\phi_{q}-\phi_{f}\right)\right]
$$

If the $V_{j r}^{*} V_{j b}$ amplitude plays the dominant role in $\overline{B_{q}^{0}} \rightarrow f$, we have

$$
\phi_{f}=2 \arg \left(V_{j r}^{*} V_{j b}\right)=\left\{\begin{array}{cc}
-2 \gamma & (j=u) \\
0 & (j=c) .
\end{array}\right.
$$

\section{Important B-Factory Benchmark Modes}

The formalism discussed in Subsection 5.3 has several interesting applications. The most important one is the extraction of the CKM angle $\beta$ from $C P$-violating effects in the "gold-plated" mode $B_{d} \rightarrow J / \psi K_{\mathrm{S}}$ [35].

\subsection{EXTRACTING $\beta$ FROM $B^{0} \rightarrow J / \psi K_{\mathrm{S}}$}

The decay $B_{d}^{0} \rightarrow J / \psi K_{\mathrm{S}}$ is a transition into a $C P$ eigenstate with eigenvalue -1 , and originates from $\bar{b} \rightarrow \bar{c} c \bar{s}$ quark-level decays. As can be seen in Fig. 10, we have to deal both with tree-diagram-like and with penguin topologies. The corresponding amplitude can be written as [36]

$$
A\left(B_{d}^{0} \rightarrow J / \psi K_{\mathrm{S}}\right)=\lambda_{c}^{(s)}\left(A_{\mathrm{cc}}^{c^{\prime}}+A_{\mathrm{pen}}^{c^{\prime}}\right)+\lambda_{u}^{(s)} A_{\mathrm{pen}}^{u^{\prime}}+\lambda_{t}^{(s)} A_{\mathrm{pen}}^{t^{\prime}},
$$



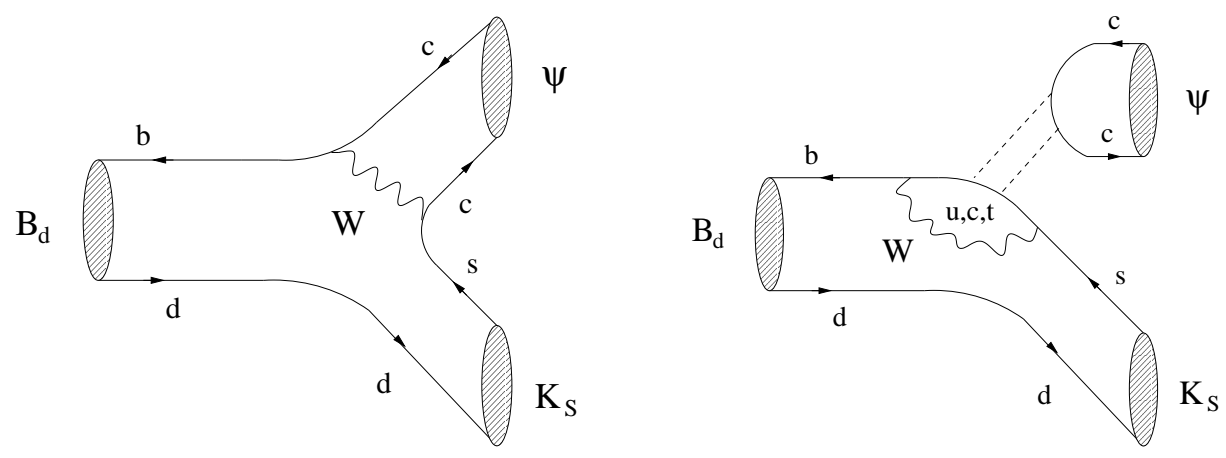

Figure 10. Feynman diagrams contributing to $B_{d}^{0} \rightarrow J / \psi K_{\mathrm{S}}$. The dashed lines in the penguin topology represent a colour-singlet exchange.

where $A_{\mathrm{cc}}^{c^{\prime}}$ denotes the current-current contributions, i.e. the "tree" processes in Fig. 10, and the strong amplitudes $A_{\mathrm{pen}}^{q^{\prime}}$ describe the contributions from penguin topologies with internal $q$ quarks $(q \in\{u, c, t\})$. These penguin amplitudes take into account both QCD and electroweak penguin contributions. The primes in (81) remind us that we are dealing with a $\bar{b} \rightarrow \bar{s}$ transition, and the

$$
\lambda_{q}^{(s)} \equiv V_{q s} V_{q b}^{*}
$$

are CKM factors. If we make use both of the unitarity of the CKM matrix, implying $\lambda_{u}^{(s)}+\lambda_{c}^{(s)}+\lambda_{t}^{(s)}=0$, and of the Wolfenstein parametrization [16], generalized to include non-leading terms in $\lambda$ [17], we obtain

$$
A\left(B_{d}^{0} \rightarrow J / \psi K_{\mathrm{S}}\right)=\left(1-\frac{\lambda^{2}}{2}\right) \mathcal{A}^{\prime}\left[1+\left(\frac{\lambda^{2}}{1-\lambda^{2}}\right) a^{\prime} e^{i \theta^{\prime}} e^{i \gamma}\right]
$$

where

$$
\mathcal{A}^{\prime} \equiv \lambda^{2} A\left(A_{\mathrm{cc}}^{c^{\prime}}+A_{\mathrm{pen}}^{c t^{\prime}}\right)
$$

with $A_{\mathrm{pen}}^{c t^{\prime}} \equiv A_{\mathrm{pen}}^{c^{\prime}}-A_{\mathrm{pen}}^{t^{\prime}}$, and

$$
a^{\prime} e^{i \theta^{\prime}} \equiv R_{b}\left(\frac{A_{\mathrm{pen}}^{u t^{\prime}}}{A_{\mathrm{cc}}^{c^{\prime}}+A_{\mathrm{pen}}^{c t^{\prime}}}\right) .
$$

The quantity $A_{\text {pen }}^{u t^{\prime}}$ is defined in analogy to $A_{\mathrm{pen}}^{c t^{\prime}}, A$ is given by (see (9))

$$
A=\left|V_{c b}\right| / \lambda^{2}=0.81 \pm 0.06,
$$

and the definition of $R_{b}=0.41 \pm 0.07$ can be found in (19).

It is very difficult to calculate the "penguin" parameter $a^{\prime} e^{i \theta^{\prime}}$, which introduces the $C P$-violating phase factor $e^{i \gamma}$ into the $B_{d}^{0} \rightarrow J / \psi K_{\mathrm{S}}$ decay 
amplitude and represents - sloppily speaking - the ratio of the penguin to tree contributions. However, this parameter - and therefore also $e^{i \gamma}$ - enters (83) in a doubly Cabibbo-suppressed way. Consequently, we have to a very good approximation $\phi_{\psi K_{\mathrm{S}}}=0$, and obtain with the help of (69) and (79):

$$
\mathcal{A}_{\mathrm{CP}}^{\mathrm{mix}}\left(B_{d} \rightarrow J / \psi K_{\mathrm{S}}\right)=+\sin \left[-\left(\phi_{d}-0\right)\right]=-\sin (2 \beta) .
$$

Since (79) applies with excellent accuracy to $B_{d} \rightarrow J / \psi K_{\mathrm{S}}$, it is referred to as the "gold-plated" mode to determine $\beta$ [35]. In addition to (87), another important implication of the Standard Model is the following relation:

$$
\mathcal{A}_{\mathrm{CP}}^{\operatorname{dir}}\left(B_{d} \rightarrow J / \psi K_{\mathrm{S}}\right) \approx 0 \approx \mathcal{A}_{\mathrm{CP}}\left(B^{+} \rightarrow J / \psi K^{+}\right) .
$$

An observation of these $C P$ asymmetries at the level of $10 \%$ would be a strong indication for new physics. There is already an interesting constraint from CLEO [37], $\mathcal{A}_{\mathrm{CP}}\left(B^{+} \rightarrow J / \psi K^{+}\right)=(-1.8 \pm 4.3 \pm 0.4) \%$, and BaBar reported $\mathcal{A}_{\mathrm{CP}}^{\text {dir }}\left(B_{d} \rightarrow J / \psi K_{\mathrm{S}}\right)=(26 \pm 19) \%$ [38].

Concerning the measurement of $\sin (2 \beta)$ through (87), there were already important first steps by the OPAL, CDF and ALEPH collaborations:

$$
\sin (2 \beta)= \begin{cases}3.2_{-2.0}^{+1.8} \pm 0.5 & (\text { OPAL [39]) } \\ 0.79_{-0.44}^{+0.41} & (\text { CDF [40]) } \\ 0.84_{-1.04}^{+0.82} \pm 0.16 & (\text { ALEPH [41]) }\end{cases}
$$

In the summer of 2000, also the first results from the asymmetric $e^{+}-e^{-}$ $B$-factories were reported:

$$
\sin (2 \beta)= \begin{cases}0.12 \pm 0.37 \pm 0.09 & (\text { BaBar }[38]) \\ 0.45_{-0.44-0.09}^{+0.43+0.07} & \text { (Belle [42]). }\end{cases}
$$

On the other hand, the CKM fits discussed in Subsection 2.4 yield the following range for the Standard-Model expectation [21]:

$$
0.53 \leq \sin (2 \beta) \leq 0.93 \text {. }
$$

Although the experimental uncertainties are still very large, the small central value reported by the BaBar collaboration [38] led already to some excitement in the $B$-physics community [43], as it would be in conflict with the Standard-Model range (91). This possible discrepancy might indicate new-physics contributions to $B_{d}^{0}-\overline{B_{d}^{0}}$ and/or $K^{0}-\overline{K^{0}}$ mixing.

After a couple of years collecting data at the $B$-factories, an experimental uncertainty of $\left.\Delta \sin (2 \beta)\right|_{\exp }=0.05$ seems to be achievable, whereas the experimental uncertainty in the LHC era is expected to be one order of magnitude higher [44]. In view of this tremendous experimental accuracy, 

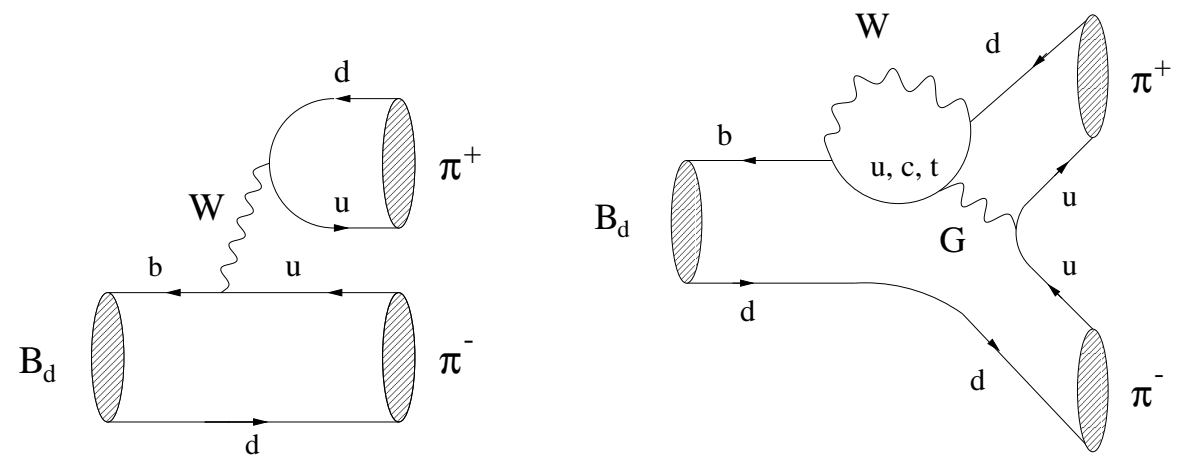

Figure 11. Feynman diagrams contributing to $B_{d}^{0} \rightarrow \pi^{+} \pi^{-}$.

it is an important issue to investigate the theoretical accuracy of (87) and (88), which is a very challenging theoretical task. An interesting channel in this respect is $B_{s} \rightarrow J / \psi K_{\mathrm{S}}$ [36], allowing us to control the - presumably very small - penguin uncertainties in the determination of $\beta$ from the $C P$-violating effects in $B_{d} \rightarrow J / \psi K_{\mathrm{S}}$, and to extract the CKM angle $\gamma$.

\subsection{PROBING $\alpha$ THROUGH $B^{0} \rightarrow \pi^{+} \pi^{-}$}

Another benchmark mode for the $B$-factories is $B_{d}^{0} \rightarrow \pi^{+} \pi^{-}$. It is a decay into a $C P$ eigenstate with eigenvalue +1 , and originates from $\bar{b} \rightarrow \bar{u} u \bar{d}$ quark-level transitions (see Fig. 11). In analogy to (81), the corresponding decay amplitude can be expressed in the following way [45]:

$$
\begin{aligned}
A\left(B_{d}^{0} \rightarrow \pi^{+} \pi^{-}\right)= & \lambda_{u}^{(d)}\left(A_{\mathrm{cc}}^{u}+A_{\mathrm{pen}}^{u}\right)+\lambda_{c}^{(d)} A_{\mathrm{pen}}^{c}+\lambda_{t}^{(d)} A_{\mathrm{pen}}^{t} \\
& =\left(1-\frac{\lambda^{2}}{2}\right) \mathcal{C}\left[e^{i \gamma}-d e^{i \theta}\right]
\end{aligned}
$$

where

$$
\mathcal{C} \equiv \lambda^{3} A R_{b}\left(A_{\mathrm{cc}}^{u}+A_{\mathrm{pen}}^{u t}\right) \quad \text { with } \quad A_{\mathrm{pen}}^{u t} \equiv A_{\mathrm{pen}}^{u}-A_{\mathrm{pen}}^{t},
$$

and

$$
d e^{i \theta} \equiv \frac{1}{\left(1-\lambda^{2} / 2\right) R_{b}}\left(\frac{A_{\mathrm{pen}}^{c t}}{A_{\mathrm{cc}}^{u}+A_{\mathrm{pen}}^{u t}}\right) .
$$

In contrast to the $B_{d}^{0} \rightarrow J / \psi K_{\mathrm{S}}$ amplitude (83), the "penguin" parameter $d e^{i \theta}$ does not enter (92) in a doubly Cabibbo-suppressed way. If we assume, for a moment, that $d=0$, the formalism discussed in Subsection 5.3 yields

$$
\mathcal{A}_{\mathrm{CP}}^{\operatorname{mix}}\left(B_{d} \rightarrow \pi^{+} \pi^{-}\right)=-\sin [-(2 \beta+2 \gamma)]=-\sin (2 \alpha),
$$




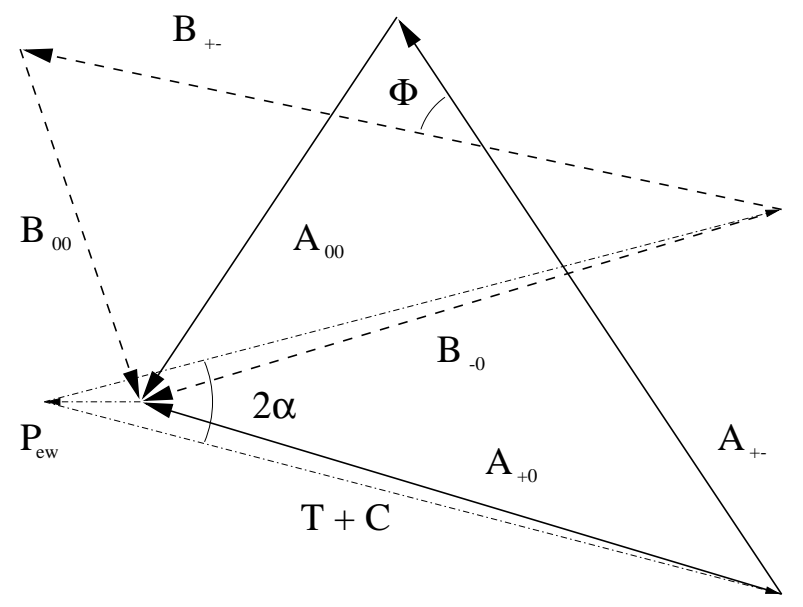

Figure 12. The $B \rightarrow \pi \pi$ isospin triangles in the complex plane. Here the amplitudes $A$ correspond to (98); the amplitudes $B$ correspond to the ones in (99), rotated by $e^{-2 i \beta}$.

which would allow a determination of $\alpha$. However, theoretical estimates typically give $d=\mathcal{O}(0.2)$, and also the present CLEO data on $B \rightarrow \pi K$ modes indicate that penguins play in fact an important role [46]. Consequently, the approximation $d=0$, i.e. the neglect of penguins in $B_{d} \rightarrow \pi^{+} \pi^{-}$, is not justified. The penguin uncertainties affecting (95) were analysed by many authors during the last couple of years [34, 47].

There are strategies to control the penguin uncertainties with the help of additional experimental data. The best known approach was proposed by Gronau and London [48], and makes use of isospin relations between the $B \rightarrow \pi \pi$ decay amplitudes. Since $B^{ \pm} \rightarrow \pi^{ \pm} \pi^{0}$ is a $\Delta I=3 / 2$ transition, the QCD penguin operators (33), which mediate $\Delta I=1 / 2$ transitions, do not contribute. Consequently, if we neglect EW penguins for a moment, we obtain

$$
A\left(B^{ \pm} \rightarrow \pi^{ \pm} \pi^{0}\right)=e^{ \pm i \gamma} e^{i \delta T+C}|T+C|
$$

which yields

$$
\frac{A\left(B^{+} \rightarrow \pi^{+} \pi^{0}\right)}{A\left(B^{-} \rightarrow \pi^{-} \pi^{0}\right)}=e^{2 i \gamma} .
$$

Moreover, the isospin symmetry implies the following amplitude relations:

$$
\begin{aligned}
& \sqrt{2} A\left(B^{+} \rightarrow \pi^{+} \pi^{0}\right)=A\left(B_{d}^{0} \rightarrow \pi^{+} \pi^{-}\right)+\sqrt{2} A\left(B_{d}^{0} \rightarrow \pi^{0} \pi^{0}\right) \\
& \sqrt{2} A\left(B^{-} \rightarrow \pi^{-} \pi^{0}\right)=A\left(\overline{B_{d}^{0}} \rightarrow \pi^{+} \pi^{-}\right)+\sqrt{2} A\left(\overline{B_{d}^{0}} \rightarrow \pi^{0} \pi^{0}\right),
\end{aligned}
$$

which can be represented as two triangles in the complex plane. These triangles can be fixed through the measured six $B \rightarrow \pi \pi$ branching ratios. In order to determine their relative orientation, we rotate the $C P$-conjugate 
triangle by $e^{-2 i \beta}$. The corresponding situation is illustrated in Fig. 12, where the angle $\Phi$ can be fixed through mixing-induced $C P$ violation [49]:

$$
\mathcal{A}_{\mathrm{CP}}^{\mathrm{mix}}\left(B_{d} \rightarrow \pi^{+} \pi^{-}\right)=-\frac{2\left|A_{+-}\right|\left|B_{+-}\right|}{\left|A_{+-}\right|^{2}+\left|B_{+-}\right|^{2}} \sin \Phi .
$$

Using (97), and taking into account that the $C P$-conjugate triangle was rotated by $e^{-2 i \beta}$, we conclude that the angle between the $B^{+} \rightarrow \pi^{+} \pi^{0}$ and $B^{-} \rightarrow \pi^{-} \pi^{0}$ amplitudes is given by $2 \alpha$. The EW penguin amplitude $P_{\mathrm{ew}}$, which was neglected so far, affects this determination of $\alpha$, as can be seen in Fig. 12. Although EW penguins play a minor role in this construction, they can be taken into account with the help of the $S U(2)$ isospin symmetry, which implies $[50,51]$

$$
\left[\frac{P_{\mathrm{ew}}}{T+C}\right]=-1.3 \times 10^{-2} \times \frac{\left|V_{t d}\right|}{\left|V_{u b}\right|} e^{i \alpha} .
$$

Unfortunately, the $B \rightarrow \pi \pi$ triangle approach is very challenging from an experimental point of view because of $\left.\mathrm{BR}\left(B_{d} \rightarrow \pi^{0} \pi^{0}\right)\right|_{\mathrm{TH}} \lesssim \mathcal{O}\left(10^{-6}\right)$. Therefore, alternative strategies are needed. An important one is provided by $B \rightarrow \rho \pi$ modes [52]. Here the final states consist of $I=0,1,2$ configurations, and the isospin symmetry implies two pentagonal relations, which correspond to (98) and (99), and also allow a determination of $\alpha$. This approach is quite complicated. However, it can be simplified by considering a maximum-likelihood fit to the parameters of the full Dalitz plot distribution, where it is assumed that the $B \rightarrow 3 \pi$ events are fully dominated by $B \rightarrow \rho \pi$ [53]. Further simplifications were proposed in Ref. [54]. An issue raised recently in this context is the impact of "polar diagrams", yielding $B \rightarrow\left\{\pi\left(B^{*}, \rho\right)\right\} \rightarrow \pi \pi \pi$ transitions. These processes may affect $B^{\mp} \rightarrow \pi^{\mp} \pi^{\mp} \pi^{ \pm}$and $B_{d} \rightarrow \rho^{0} \pi^{0}$, and represent an irreducible background in the Dalitz plot [55].

Another possibility to eliminate the penguin uncertainties in the extraction of $\alpha$ from $B_{d} \rightarrow \pi^{+} \pi^{-}$is to combine this channel with $B_{d} \rightarrow K^{0} \overline{K^{0}}$ through the $S U(3)$ flavour symmetry [56]. A simple strategy to extract $\alpha$, making also use of $S U(3)$, was proposed in [57]; refinements of this approach and further interesting methods were suggested in [58].

Let us finally note that a particularly interesting strategy is provided by the decay $B_{s} \rightarrow K^{+} K^{-}$, which is related to $B_{d} \rightarrow \pi^{+} \pi^{-}$by interchanging all down and strange quarks, i.e. through the $U$-spin flavour symmetry of strong interactions. A combined analysis of these two channels allows a simultaneous determination of $\beta$ and $\gamma$ [45], which has certain theoretical advantages, appears to be promising for CDF-II [59], and is ideally suited for $\mathrm{LHCb}$ [44]. This approach is discussed in detail in Section 10. 

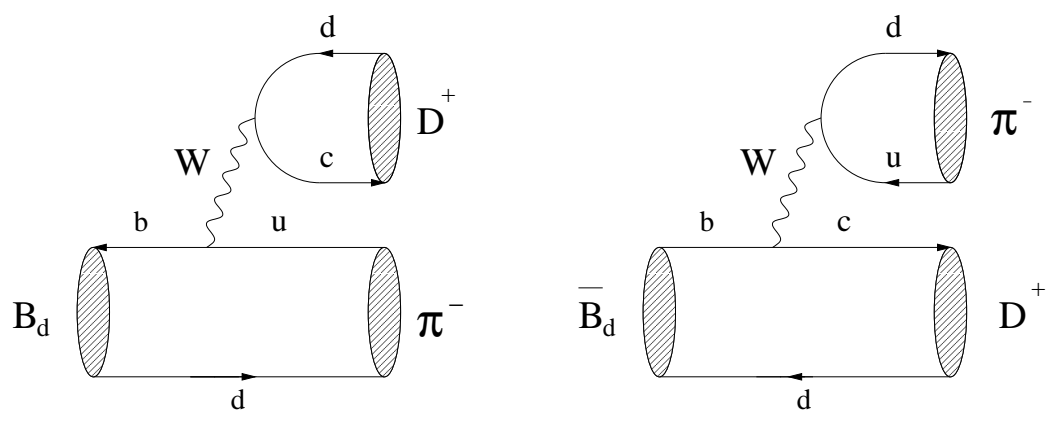

Figure 13. Feynman diagrams contributing to $B_{d}^{0}, \overline{B_{d}^{0}} \rightarrow D^{(*)+} \pi^{-}$.

\subsection{EXTRACTING $2 \beta+\gamma$ FROM $B^{0} \rightarrow D^{(*) \pm} \pi^{\mp}$ DECAYS}

So far, we have put a strong emphasis on neutral $B$ decays into final $C P$ eigenstates. However, in order to extract CKM phases, there are also interesting decays of $B_{d, s}$ mesons into final states that are not eigenstates of the $C P$ operator. An important example is given by $B_{d} \rightarrow D^{(*) \pm} \pi^{\mp}$ decays, which receive only contributions from tree-diagram-like topologies. As can be seen in Fig. $13, B_{d^{-}}^{0}$ and $\overline{B_{d}^{0}}$-mesons may both decay into $D^{(*)+} \pi^{-}$, thereby leading to interference effects between $B_{d}^{0}-\overline{B_{d}^{0}}$ mixing and decay processes. Consequently, the time-dependent rates for decays of initially, i.e. at time $t=0$, present $B_{d^{-}}^{0}$ or $\overline{B_{d}^{0}}$-mesons into the final state $f \equiv D^{(*)+} \pi^{-}$ (see (65)) allow us to determine the observable [30]

$$
\xi_{f}^{(d)}=e^{-i \Theta_{M_{12}(d)}} \frac{A\left(\overline{B_{d}^{0}} \rightarrow f\right)}{A\left(B_{d}^{0} \rightarrow f\right)}=-e^{-i\left(\phi_{d}+\gamma\right)}\left(\frac{1-\lambda^{2}}{\lambda^{2} R_{b}}\right) \frac{\bar{M}_{f}}{M_{\bar{f}}},
$$

whereas those corresponding to $\bar{f} \equiv D^{(*)-} \pi^{+}$provide

$$
\xi_{\bar{f}}^{(d)}=e^{-i \Theta_{M_{12}}^{(d)}} \frac{A\left(\overline{B_{d}^{0}} \rightarrow \bar{f}\right)}{A\left(B_{d}^{0} \rightarrow \bar{f}\right)}=-e^{-i\left(\phi_{d}+\gamma\right)}\left(\frac{\lambda^{2} R_{b}}{1-\lambda^{2}}\right) \frac{M_{\bar{f}}}{\bar{M}_{f}} .
$$

Here, $R_{b}$ is the usual CKM factor (see (19)), and

$$
\begin{aligned}
\bar{M}_{f} & \equiv\left\langle f\left|\overline{\mathcal{O}}_{1}(\mu) \mathcal{C}_{1}(\mu)+\overline{\mathcal{O}}_{2}(\mu) \mathcal{C}_{2}(\mu)\right| \overline{B_{d}^{0}}\right\rangle \\
M_{\bar{f}} & \equiv\left\langle\bar{f}\left|\mathcal{O}_{1}(\mu) \mathcal{C}_{1}(\mu)+\mathcal{O}_{2}(\mu) \mathcal{C}_{2}(\mu)\right| \overline{B_{d}^{0}}\right\rangle
\end{aligned}
$$

are hadronic matrix elements of the following current-current operators:

$$
\begin{aligned}
& \overline{\mathcal{O}}_{1}=\left(\bar{d}_{\alpha} u_{\beta}\right)_{\mathrm{V}-\mathrm{A}}\left(\bar{c}_{\beta} b_{\alpha}\right)_{\mathrm{V}-\mathrm{A}}, \quad \overline{\mathcal{O}}_{2}=\left(\bar{d}_{\alpha} u_{\alpha}\right)_{\mathrm{V}-\mathrm{A}}\left(\bar{c}_{\beta} b_{\beta}\right)_{\mathrm{V}-\mathrm{A}}, \\
& \mathcal{O}_{1}=\left(\bar{d}_{\alpha} c_{\beta}\right)_{\mathrm{V}-\mathrm{A}}\left(\bar{u}_{\beta} b_{\alpha}\right)_{\mathrm{V}-\mathrm{A}}, \quad \mathcal{O}_{2}=\left(\bar{d}_{\alpha} c_{\alpha}\right)_{\mathrm{V}-\mathrm{A}}\left(\bar{u}_{\beta} b_{\beta}\right)_{\mathrm{V}-\mathrm{A}},
\end{aligned}
$$


which are completely analogous to the ones we encountered in the discussion of $\overline{B_{d}^{0}} \rightarrow D^{+} K^{-}$in Subsection 4.2. The observables $\xi_{f}^{(d)}$ and $\xi_{\bar{f}}^{(d)}$ allow a theoretically clean extraction of the weak phase $\phi_{d}+\gamma$ [60], as the hadronic matrix elements $\bar{M}_{f}$ and $M_{\bar{f}}$ cancel in the following combination:

$$
\xi_{f}^{(d)} \times \xi_{\bar{f}}^{(d)}=e^{-2 i\left(\phi_{d}+\gamma\right)} .
$$

Since $\phi_{d}$, i.e. $2 \beta$, can be determined straightforwardly with the help of the "gold-plated" mode $B_{d} \rightarrow J / \psi K_{\mathrm{S}}$ (see Subsection 6.1), we may extract the CKM angle $\gamma$ from (107). As the $\bar{b} \rightarrow \bar{u}$ quark-level transition in Fig. 13 is doubly Cabibbo-suppressed by $\lambda^{2} R_{b} \approx 0.02$ with respect to the $b \rightarrow c$ transition, the interference effects are tiny. However, the branching ratios are large, i.e. of order $10^{-3}$, and the $D^{(*) \pm} \pi^{\mp}$ states can be reconstructed with a good efficiency and modest backgrounds. Consequently, $B_{d} \rightarrow D^{(*) \pm} \pi^{\mp}$ decays offer an interesting strategy to determine $\gamma$. Experimental feasibility studies can be found in Refs. [44, 61].

\section{CP Violation in Charged B Decays}

Since there are no mixing effects present in the charged $B$-meson system, non-vanishing $C P$ asymmetries of the kind

$$
\mathcal{A}_{\mathrm{CP}} \equiv \frac{\Gamma\left(B^{+} \rightarrow f\right)-\Gamma\left(B^{-} \rightarrow \bar{f}\right)}{\Gamma\left(B^{+} \rightarrow f\right)+\Gamma\left(B^{-} \rightarrow \bar{f}\right)}
$$

would give us unambiguous evidence for "direct" $C P$ violation in the $B$ system, similarly as $\operatorname{Re}\left(\varepsilon^{\prime} / \varepsilon\right) \neq 0$ does in the neutral kaon system. The $C P$ asymmetries (108), which correspond to $\mathcal{A}_{\mathrm{CP}}^{\mathrm{dir}}\left(B_{q} \rightarrow f\right)$ in (68), arise from interference between decay amplitudes with different $C P$-violating weak and $C P$-conserving strong phases. Because of the unitarity of the CKM matrix, any $B$-decay amplitude can be expressed within the Standard Model in the following way:

$$
A\left(B^{ \pm} \rightarrow f\right)=\left|A_{1}\right| e^{i \delta_{1}} e^{ \pm i \varphi_{1}}+\left|A_{2}\right| e^{i \delta_{2}} e^{ \pm i \varphi_{2}} .
$$

Here the $\delta_{1,2}$ are $C P$-conserving strong phases, which are induced by finalstate-interaction (FSI) processes, whereas the $\varphi_{1,2}$ are $C P$-violating weak phases, which originate from the CKM matrix. Using (109), we obtain

$$
\mathcal{A}_{\mathrm{CP}}=\frac{-2\left|A_{1}\right|\left|A_{2}\right| \sin \left(\varphi_{1}-\varphi_{2}\right) \sin \left(\delta_{1}-\delta_{2}\right)}{\left|A_{1}\right|^{2}+2\left|A_{1}\right|\left|A_{2}\right| \cos \left(\varphi_{1}-\varphi_{2}\right) \cos \left(\delta_{1}-\delta_{2}\right)+\left|A_{2}\right|^{2}} .
$$

Consequently, a non-vanishing direct $C P$ asymmetry $\mathcal{A}_{\mathrm{CP}}$ requires both a non-trivial strong and a non-trivial weak phase difference. In addition to 

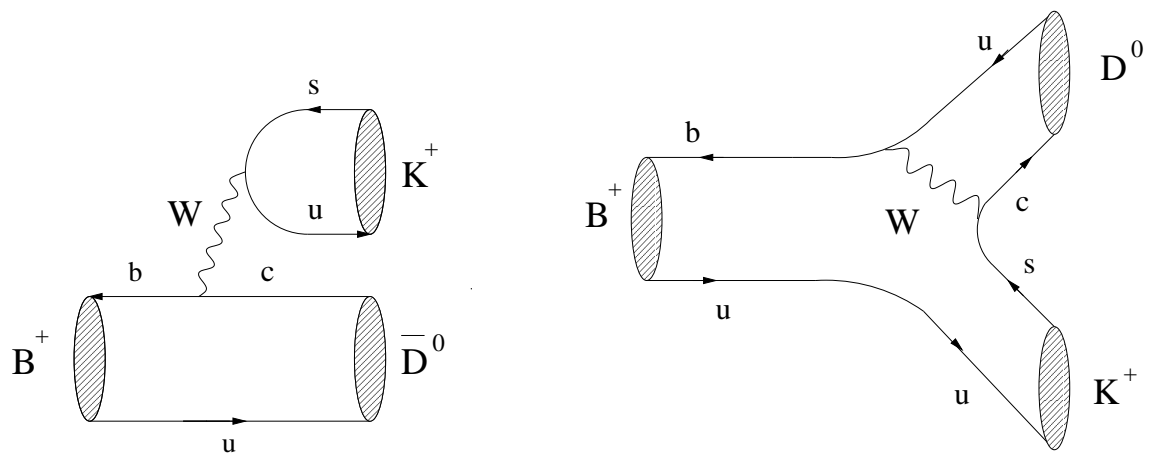

Figure 14. Feynman diagrams contributing to $B^{+} \rightarrow \overline{D^{0}} K^{+}$and $B^{+} \rightarrow D^{0} K^{+}$.

the hadronic amplitudes $\left|A_{1,2}\right|$, the strong phases $\delta_{1,2}$ lead to particularly large hadronic uncertainties in (110), thereby destroying the clean relation to the $C P$-violating weak phase $\varphi_{1}-\varphi_{2}$.

\subsection{EXTRACTING $\gamma$ FROM $B^{ \pm} \rightarrow K^{ \pm} D$ DECAYS}

An important tool to eliminate the hadronic uncertainties in charged $B$ decays is given by amplitude relations. The prototype of this approach, which was proposed by Gronau and Wyler [62], uses $B^{ \pm} \rightarrow K^{ \pm} D$ decays and allows a theoretically clean extraction of $\gamma$. The decays $B^{+} \rightarrow \overline{D^{0}} K^{+}$ and $B^{+} \rightarrow D^{0} K^{+}$are pure "tree" decays, as can be seen in Fig. 14. If we make, in addition, use of the transition $B^{+} \rightarrow D_{+}^{0} K^{+}$, where $D_{+}^{0}$ denotes the $C P$ eigenstate of the neutral $D$-meson system with $C P$ eigenvalue +1 ,

$$
\left|D_{+}^{0}\right\rangle=\frac{1}{\sqrt{2}}\left(\left|D^{0}\right\rangle+\left|\overline{D^{0}}\right\rangle\right)
$$

we obtain

$$
\begin{aligned}
& \sqrt{2} A\left(B^{+} \rightarrow K^{+} D_{+}^{0}\right)=A\left(B^{+} \rightarrow K^{+} D^{0}\right)+A\left(B^{+} \rightarrow K^{+} \overline{D^{0}}\right) \\
& \sqrt{2} A\left(B^{-} \rightarrow K^{-} D_{+}^{0}\right)=A\left(B^{-} \rightarrow K^{-} \overline{D^{0}}\right)+A\left(B^{-} \rightarrow K^{-} D^{0}\right) .
\end{aligned}
$$

These relations can be represented as two triangles in the complex plane. As we have only to deal with tree-diagram-like topologies, we have moreover

$$
\begin{aligned}
A \equiv A\left(B^{+} \rightarrow K^{+} \overline{D^{0}}\right) & =A\left(B^{-} \rightarrow K^{-} D^{0}\right) \\
a \equiv A\left(B^{+} \rightarrow K^{+} D^{0}\right) & =A\left(B^{-} \rightarrow K^{-} \overline{D^{0}}\right) \times e^{2 i \gamma}
\end{aligned}
$$

allowing a theoretically clean extraction of $\gamma$ (see Fig. 15) [62]. Unfortunately, the triangles are very squashed ones, since $a \equiv A\left(B^{+} \rightarrow K^{+} D^{0}\right)$ is 


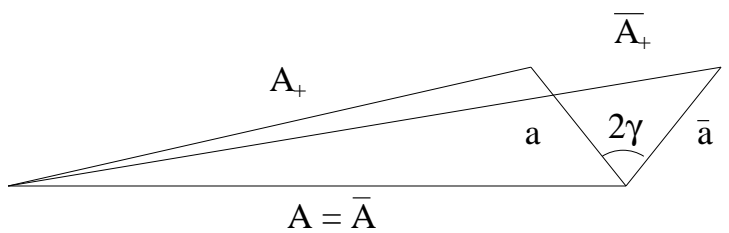

Figure 15. Triangle relations between charged $B^{ \pm} \rightarrow K^{ \pm} D$ decay amplitudes.

colour-suppressed with respect to $A \equiv A\left(B^{+} \rightarrow K^{+} \overline{D^{0}}\right)$ :

$$
\frac{|a|}{|A|}=\frac{|\bar{a}|}{|\bar{A}|} \approx \frac{1}{\lambda} \frac{\left|V_{u b}\right|}{\left|V_{c b}\right|} \times \frac{a_{2}}{a_{1}} \approx 0.41 \times \frac{a_{2}}{a_{1}} \approx 0.1 .
$$

Consequently, the $B^{ \pm} \rightarrow K^{ \pm} D$ approach is very difficult from an experimental point of view (see also [63]). As an alternative, the decays $B_{d} \rightarrow K^{* 0} D$ were proposed, where the triangles are more equilateral [64]. But all sides are small (colour-suppressed) so that these decays are also not perfectly suited for the "triangle" approach.

\subsection{EXTRACTING $\gamma$ FROM $B_{C}^{ \pm} \rightarrow D_{S}^{ \pm} D$ DECAYS}

The decays $B_{c}^{ \pm} \rightarrow D_{s}^{ \pm} D$ are the $B_{c}$-meson counterparts of $B_{u}^{ \pm} \rightarrow K^{ \pm} D$ and also allow an extraction of $\gamma$ [65], which relies on the amplitude relations

$$
\begin{aligned}
& \sqrt{2} A\left(B_{c}^{+} \rightarrow D_{s}^{+} D_{+}^{0}\right)=A\left(B_{c}^{+} \rightarrow D_{s}^{+} D^{0}\right)+A\left(B_{c}^{+} \rightarrow D_{s}^{+} \overline{D^{0}}\right) \\
& \sqrt{2} A\left(B_{c}^{-} \rightarrow D_{s}^{-} D_{+}^{0}\right)=A\left(B_{c}^{-} \rightarrow D_{s}^{-} \overline{D^{0}}\right)+A\left(B_{c}^{-} \rightarrow D_{s}^{-} D^{0}\right),
\end{aligned}
$$

with

$$
\begin{aligned}
& A\left(B_{c}^{+} \rightarrow D_{s}^{+} \overline{D^{0}}\right)=A\left(B_{c}^{-} \rightarrow D_{s}^{-} D^{0}\right) \\
& A\left(B_{c}^{+} \rightarrow D_{s}^{+} D^{0}\right)=A\left(B_{c}^{-} \rightarrow D_{s}^{-} \overline{D^{0}}\right) \times e^{2 i \gamma} .
\end{aligned}
$$

At first sight, everything is completely analogous to $B^{ \pm} \rightarrow K^{ \pm} D$. However, there is an important difference [66]: in the $B_{c}^{ \pm} \rightarrow D_{s}^{ \pm} D$ system, the amplitude with the rather small CKM matrix element $V_{u b}$ is not colour suppressed, while the larger element $V_{c b}$ comes with a colour-suppression factor. Therefore, we obtain

$$
\left|\frac{A\left(B_{c}^{+} \rightarrow D_{s}^{+} D^{0}\right)}{A\left(B_{c}^{+} \rightarrow D_{s}^{+} \overline{D^{0}}\right)}\right| \approx \frac{1}{\lambda} \frac{\left|V_{u b}\right|}{\left|V_{c b}\right|} \times \frac{a_{1}}{a_{2}} \approx 0.41 \times \frac{a_{1}}{a_{2}}=\mathcal{O}(1),
$$

and conclude that the two amplitudes are similar in size. Decays of the type $B_{c}^{ \pm} \rightarrow D^{ \pm} D$ - the $U$-spin counterparts of $B_{c}^{ \pm} \rightarrow D_{s}^{ \pm} D$ - can be 
added to the analysis, as well as channels, where the $D_{s}^{ \pm}$- and $D^{ \pm}$-mesons are replaced by higher resonances. At the LHC, one expects about $10^{10}$ untriggered $B_{c}$-mesons per year of running. Provided there are no serious experimental problems, the $B_{c}^{ \pm} \rightarrow D_{(s)}^{ \pm} D$ approach should be interesting for the $B$-physics programme of the LHC. From a theoretical point of view, it is the ideal realization of the "triangle" approach to extract $\gamma$. In Section 9, we will discuss other strategies to extract $\gamma$, making use of amplitude relations between $B \rightarrow \pi K$ decays.

\section{The "El Dorado" for Hadron Machines: the $B_{s}$ System}

Unfortunately, at the $e^{+}-e^{-} B$-factories operating at the $\Upsilon(4 S)$ resonance, no $B_{s}$-mesons are accessible. On the other hand, $B_{s}$ decays are very promising for hadron machines, where plenty of $B_{s}$-mesons can be produced. There are important differences between the $B_{d}$ and $B_{s}$ systems:

- Within the Standard Model, the $B_{s}^{0}-\overline{B_{s}^{0}}$ mixing phase is negligibly small, $\phi_{s}=-2 \lambda^{2} \eta=\mathcal{O}(0.03)$, whereas $\phi_{d}=2 \beta=\mathcal{O}\left(50^{\circ}\right)$.

- A large mixing parameter $x_{s}=\mathcal{O}(20)$ is expected in the Standard Model (see (64)), whereas $x_{d}=0.723 \pm 0.032$. The present experimental lower bound is $\Delta M_{s}>15.0 \mathrm{ps}^{-1}, x_{s}>21.3$ (95\% C.L.) [67].

- There may be a sizeable width difference $\Delta \Gamma_{s} / \Gamma_{s}=\mathcal{O}(10 \%)$, whereas $\Delta \Gamma_{d}$ is negligibly small. The present CDF and LEP average is given by $\Delta \Gamma_{s} / \Gamma_{s}=0.16_{-0.13}^{+0.16}, \Delta \Gamma_{s} / \Gamma_{s}<0.31$ (95\% C.L.) [68].

\section{1. $\Delta M_{S}$ AND CONSTRAINTS IN THE $\bar{\rho}-\bar{\eta}$ PLANE}

As we have noted in Subsection 2.4, the mass difference $\Delta M_{d}$ plays an important role to constrain the apex of the unitarity triangle shown in Fig. 2 (a). In particular, it allows us to fix a circle in the $\bar{\rho}-\bar{\eta}$ plane around $(1,0)$ with radius $R_{t}$. Concerning the theoretical uncertainties, it is -instead of using $\Delta M_{d}$ separately - more advantageous to use the ratio

$$
\frac{\left|V_{t d}\right|}{\left|V_{t s}\right|}=\xi \sqrt{\frac{m_{B_{s}}}{m_{B_{d}}}} \sqrt{\frac{\Delta M_{d}}{\Delta M_{s}}},
$$

where the $S U(3)$-breaking parameter

$$
\xi \equiv \frac{F_{B_{s}} \sqrt{B_{B_{s}}}}{F_{B_{d}} \sqrt{B_{B_{d}}}}=1.16 \pm 0.07
$$

can be determined with the help of lattice or QCD sum rule calculations. Interestingly, the presently available experimental lower bound on $\Delta M_{s}$ can 


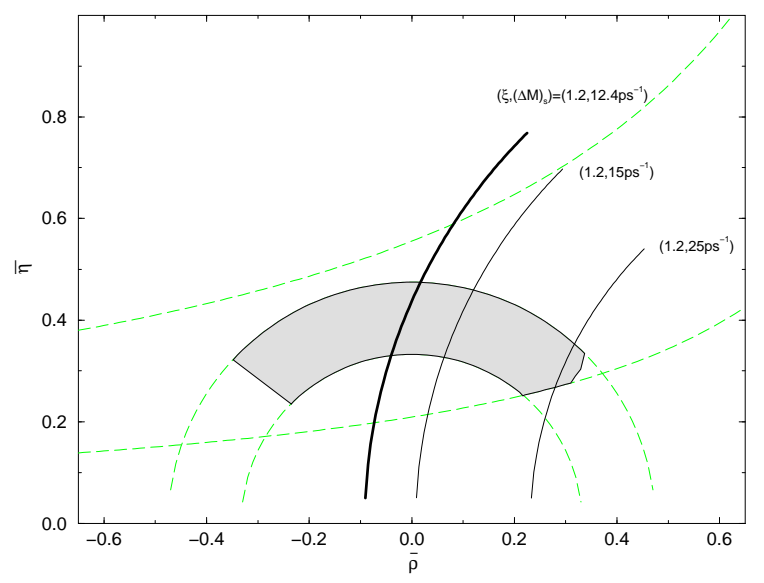

Figure 16. The impact of the upper limit $\left(R_{t}\right)_{\max }$ on the allowed range in the $\bar{\rho}-\bar{\eta}$ plane.

be transformed into an upper bound on $R_{t}$ [69]:

$$
\left(R_{t}\right)_{\max }=1.0 \times \xi \times \sqrt{\frac{10.2 / \mathrm{ps}}{\left(\Delta M_{s}\right)_{\min }}} .
$$

In Fig. 16, we show the impact of this relation on the allowed range in the $\bar{\rho}-\bar{\eta}$ plane [4]. The strong present lower bound on $\Delta M_{s}$ excludes already a large part in the $\bar{\rho}-\bar{\eta}$ plane, implying in particular $\gamma<90^{\circ}$.

\section{2. $\Delta \Gamma_{S}$ AND UNTAGGED DECAY RATES}

A non-vanishing width difference $\Delta \Gamma_{s}$ may allow the extraction of $C P$ violating weak phases from the following "untagged" $B_{s}$ rates [70]-[74]:

$$
\Gamma[f(t)] \equiv \Gamma\left(B_{s}^{0}(t) \rightarrow f\right)+\Gamma\left(\overline{B_{s}^{0}}(t) \rightarrow f\right) .
$$

Using (65), we obtain

$$
\begin{aligned}
\Gamma[f(t)] \propto & {\left[\left(1+\left|\xi_{f}^{(s)}\right|^{2}\right)\left(e^{-\Gamma_{\mathrm{L}}^{(s)} t}+e^{-\Gamma_{\mathrm{H}}^{(s)} t}\right)\right.} \\
& \left.-2 \operatorname{Re} \xi_{f}^{(s)}\left(e^{-\Gamma_{\mathrm{L}}^{(s)} t}-e^{-\Gamma_{\mathrm{H}}^{(s)}} t\right)\right],
\end{aligned}
$$

where the $\Delta M_{s} t$ terms cancel. Because of the large mixing parameter $x_{s}$, the $\Delta M_{s} t$ terms arising in "tagged" rates oscillate very rapidly and are hard to resolve. Although it should be not problem to accomplish this task at the LHC, studies of untagged rates are interesting in terms of efficiency, acceptance and purity. Moreover, if $\Delta \Gamma_{s}$ is sizeable, we may extract the 
observable $\mathcal{A}_{\Delta \Gamma}\left(B_{s} \rightarrow f\right)$ (see (70)) from (126). Still it is not clear whether $\Delta \Gamma_{s}$ is large enough to make this possible. Concerning the theoretical status of $\Delta \Gamma_{s}$, next-to-leading order QCD corrections were calculated using the heavy-quark expansion [75]; the results for $\Delta \Gamma_{s}$ depend crucially on the size of the relevant hadronic matrix elements. The authors of Ref. [76] find $\Delta \Gamma_{s} / \Gamma_{s}=(10.7 \pm 2.6 \pm 1.4 \pm 1.7) \%$, whereas another group [77] gives the smaller value $(4.7 \pm 1.5 \pm 1.6) \%$. The difference between these results is mainly related to the $B_{s}$ decay constant $F_{B_{s}}$. Besides "unquenching", a better determination of $1 / m_{b}$ corrections is very important to reduce the uncertainties of these lattice calculations.

\subsection{PURE TREE DECAYS}

An interesting class of $B_{s}$ decays is due to $b \rightarrow c \bar{u} s$ quark-level transitions, providing the $B_{s}$ variant of the $B_{d} \rightarrow D^{(*) \pm} \pi^{\mp}$ approach to extract $\gamma+2 \beta$ discussed in Subsection 6.3. Here we have also to deal with pure "tree" decays, where both $B_{s}^{0}$ - and $\overline{B_{s}^{0}}$-mesons may decay into the same final state $f$. The resulting interference effects between decay and mixing processes allow a theoretically clean extraction of $\gamma-2 \delta \gamma$ from

$$
\xi_{f}^{(s)} \times \xi_{\bar{f}}^{(s)}=e^{-2 i(\gamma-2 \delta \gamma)},
$$

where the $B_{s}^{0}-\overline{B_{s}^{0}}$ mixing phase $-2 \delta \gamma$ is negligibly small in the Standard Model. It can be probed through $C P$-violating effects in $B_{s} \rightarrow J / \psi \phi$, as we will see below. An interesting difference to the $B_{d} \rightarrow D^{(*) \pm} \pi^{\mp}$ strategy is that both decay paths of $B_{s}^{0}, \overline{B_{s}^{0}} \rightarrow f$ are of the same order of $\lambda$, thereby leading to larger interference effects.

There are several well-known strategies making use of these features: we may consider the colour-allowed decays $B_{s} \rightarrow D_{s}^{ \pm} K^{\mp}[78]$, or the coloursuppressed modes $B_{s} \rightarrow D^{0} \phi$ [79]. Strategies employing "untagged" $B_{s}$ decays were also proposed, where the width difference $\Delta \Gamma_{s}$ and the angular distributions of $B_{s} \rightarrow D_{s}^{* \pm} K^{* \mp}$ or $B_{s} \rightarrow D^{* 0} \phi$ channels play a key role [72] (see also [80]). Recently, strategies using "CP-tagged" $B_{s}$ decays were proposed [81], which require a symmetric $e^{+}-e^{-}$collider operated at the $\Upsilon(5 S)$ resonance. In this approach, initially present $C P$ eigenstates $B_{s}^{C P}$ are employed, which can be tagged by making use of the fact that the $B_{s}^{0} / \overline{B_{s}^{0}}$ mixtures have anti-correlated $C P$ eigenvalues at $\Upsilon(5 S)$.

\subsection{A CLOSER LOOK AT $B_{S} \rightarrow J / \psi \phi$}

This decay is the $B_{s}$ counterpart to $B_{d} \rightarrow J / \psi K_{\mathrm{S}}$ and offers interesting strategies to extract $\Delta M_{s}$ and $\Delta \Gamma_{s}$, and to probe $\phi_{s}=-2 \delta \gamma$ [82]. The corresponding Feynman diagrams are completely analogous to those shown in 


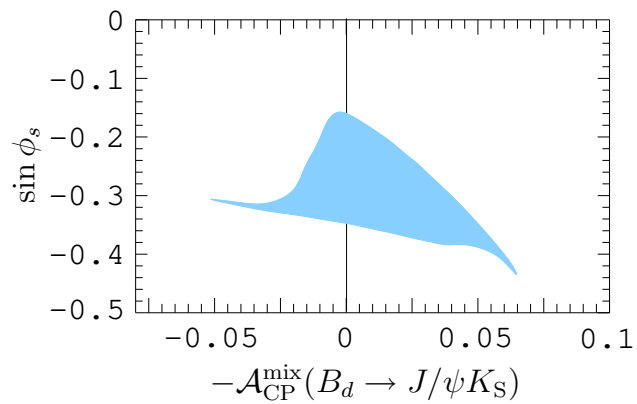

Figure 17. The allowed region for $\mathcal{A}_{\mathrm{CP}}^{\mathrm{mix}}\left(B_{d} \rightarrow J / \psi K_{\mathrm{S}}\right)$ and $\sin \phi_{s}$ in the SB-LR model.

Fig. 10. Since the final state of $B_{s} \rightarrow J / \psi \phi$ is an admixture of different $C P$ eigenstates, we have to use the angular distribution of the $J / \psi \rightarrow l^{+} l^{-}$and $\phi \rightarrow K^{+} K^{-}$decay products to disentangle them [83]. The corresponding observables are governed by

$$
\xi_{\psi \phi}^{(s)} \propto e^{-i \phi_{s}}\left[1-2 i \sin \gamma \times \mathcal{O}\left(10^{-3}\right)\right],
$$

where the $\mathcal{O}\left(10^{-3}\right)$ factor is an abbreviation for $\left|\lambda_{u}^{(s)} \tilde{A}_{\mathrm{pen}}^{u t^{\prime}}\right| /\left|\lambda_{c}^{(s)}\left(\tilde{A}_{\mathrm{cc}}^{c^{\prime}}+\tilde{A}_{\mathrm{pen}}^{c c^{\prime}}\right)\right|$ [44]. Since $\phi_{s}=-2 \lambda^{2} \eta=\mathcal{O}(0.03)$ in the Standard Model, there may well be hadronic uncertainties as large as $\mathcal{O}(10 \%)$ in the extraction of $\phi_{s}$ form the $B_{s} \rightarrow J / \psi\left[\rightarrow l^{+} l^{-}\right] \phi\left[\rightarrow K^{+} K^{-}\right]$angular distribution, which may be an important issue in the LHC era. These hadronic uncertainties can be controlled with the help of the decay $B_{d} \rightarrow J / \psi \rho^{0}$, which has also some other interesting features [84].

It is an important implication of (128) that the $C P$-violating effects exhibited by $B_{s} \rightarrow J / \psi \phi$ are very small in the Standard Model, thereby making this channel an interesting probe to search for new physics [85]. A particular scenario for physics beyond the Standard Model, the symmetrical $S U_{\mathrm{L}}(2) \times S U_{\mathrm{R}}(2) \times U(1)$ model with spontaneous $C P$ violation $(\mathrm{SB}-\mathrm{LR})$ [86, 87], was considered in Ref. [88] to illustrate this feature. Needless to note, there are also other scenarios for new physics which are interesting in this respect, for example models allowing mixing to a new isosinglet down quark, as in $E_{6}$ [89]. In the SB-LR model, we obtain the allowed region for the mixing-induced $C P$ asymmetry in $B_{d} \rightarrow J / \psi K_{\mathrm{S}}$ and for $\sin \phi_{s}$ shown in Fig. 17 [88]. Here $\phi_{s}=\phi_{s}^{\mathrm{SM}}+\phi_{s}^{\mathrm{NP}}=-2 \lambda^{2} \eta+\phi_{s}^{\mathrm{NP}}$, where $\phi_{s}^{\mathrm{NP}}$ originates from new physics. The quantity $\sin \phi_{s}$ governs $C P$ violation in $B_{s} \rightarrow J / \psi \phi$ :

$$
\frac{\Gamma(t)-\bar{\Gamma}(t)}{\Gamma(t)+\bar{\Gamma}(t)}=\left[\frac{1-D}{F_{+}(t)+D F_{-}(t)}\right] \sin \left(\Delta M_{s} t\right) \sin \phi_{s}
$$




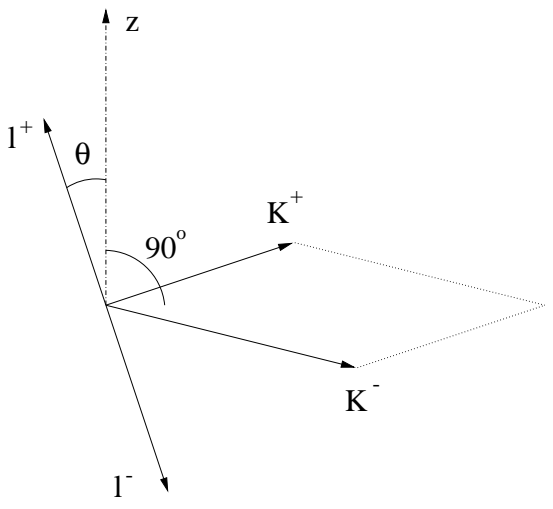

Figure 18. The kinematics of $B_{s} \rightarrow J / \psi\left[\rightarrow l^{+} l^{-}\right] \phi\left[\rightarrow K^{+} K^{-}\right]$.

where $\Gamma(t)$ and $\bar{\Gamma}(t)$ denote the time-dependent rates for decays of initially, i.e. at $t=0$, present $B_{s}^{0}$ - and $\overline{B_{s}^{0}}$-mesons into $J / \psi \phi$ final states, respectively,

$$
D \equiv \frac{\left|A_{\perp}(0)\right|^{2}}{\left|A_{0}(0)\right|^{2}+\left|A_{\|}(0)\right|^{2}}=0.1 \ldots 0.5
$$

is a hadronic factor involving linear polarization amplitudes ${ }^{3}$ and

$$
F_{ \pm}(t) \equiv \frac{1}{2}\left[\left(1 \pm \cos \phi_{s}\right) e^{+\Delta \Gamma_{s} t / 2}+\left(1 \mp \cos \phi_{s}\right) e^{-\Delta \Gamma_{s} t / 2}\right] .
$$

The range given in (130) corresponds to "factorization" [82], and is in agreement with a recent analysis of the $B_{s} \rightarrow J / \psi \phi$ polarization amplitudes $A_{0}(0), A_{\|}(0), A_{\perp}(0)$ performed by the CDF collaboration [90].

If we look at Fig. 17, we observe that $\left|\sin \phi_{s}\right|$ may be as large as 0.4 in the SB-LR model, in contrast to its vanishingly small value in the Standard Model. Another interesting feature of this figure is the preferred small value for $-\mathcal{A}_{\mathrm{CP}}^{\operatorname{mix}}\left(B_{d} \rightarrow J / \psi K_{\mathrm{S}}\right)=\sin \phi_{d}$. As we have noted in Subsection 6.1, the first result for this observable reported by the BaBar collaboration (see (90)) may point towards such a situation. Consequently, it would be very exciting to measure also (129), and to explore whether the pattern of small $C P$ violation in $B_{d} \rightarrow J / \psi K_{\mathrm{S}}$ and large $C P$ violation in $B_{s} \rightarrow J / \psi \phi$ is actually realized in nature [88].

The $C P$ asymmetry (129) may provide a "smoking-gun" signal for new physics, although it does not allow a clean determination of $\sin \phi_{s}$ because of the hadronic parameter $D$. In order to solve this problem, an angular analysis of the $B_{s} \rightarrow J / \psi\left[\rightarrow l^{+} l^{-}\right] \phi\left[\rightarrow K^{+} K^{-}\right]$decay products has to

\footnotetext{
${ }^{3} A_{\perp}$ and $A_{0}, A_{\|}$correspond to $C P$-odd and $C P$-even configurations, respectively.
} 
TABLE $1 .\langle\mathrm{BR}\rangle \mathrm{s}$ in $10^{-6}$ units; the $C P$ asymmetries $\mathcal{A}_{\mathrm{CP}}$ are from CLEO.

\begin{tabular}{ccccc}
\hline Decay & CLEO & BaBar & Belle & $\mathcal{A}_{\mathrm{CP}} / 10^{-2}$ \\
\hline$B_{d} \rightarrow \pi^{\mp} K^{ \pm}$ & $17.2_{-2.4}^{+2.5} \pm 1.2$ & $12.5_{-2.6-1.7}^{+3.0+1.3}$ & $17.4_{-4.6}^{+5.1} \pm 3.4$ & $0.04 \pm 0.16$ \\
$B^{ \pm} \rightarrow \pi^{0} K^{ \pm}$ & $11.6_{-2.7-1.3}^{+3.0+1.4}$ & & $18.8_{-4.9}^{+5.5} \pm 2.3$ & $0.29 \pm 0.23$ \\
$B^{ \pm} \rightarrow \pi^{ \pm} K$ & $18.2_{-4.0}^{+4.6} \pm 1.6$ & & & $-0.18 \pm 0.24$ \\
$B_{d} \rightarrow \pi^{0} K$ & $14.6_{-5.1-3.3}^{+5.9+2.4}$ & & $21_{-7.8-2.3}^{+9.3+2.5}$ & \\
\hline
\end{tabular}

be performed. As the full three-angle distribution is quite complicated [82], let us consider here the one-angle distribution:

$$
\frac{d \Gamma(t)}{d \cos \Theta} \propto\left(\left|A_{0}(t)\right|^{2}+\left|A_{\|}(t)\right|^{2}\right) \frac{3}{8}\left(1+\cos ^{2} \Theta\right)+\left|A_{\perp}(t)\right|^{2} \frac{3}{4} \sin ^{2} \Theta,
$$

where the kinematics is shown in Fig. 18, corresponding to the $J / \psi$ rest frame. The one-angle distribution (132) allows us to extract the observables

$$
P_{+}(t) \equiv\left|A_{0}(t)\right|^{2}+\left|A_{\|}(t)\right|^{2}, \quad P_{-}(t) \equiv\left|A_{\perp}(t)\right|^{2},
$$

as well as their $C P$ conjugates, thereby providing the $C P$ asymmetries

$$
\frac{P_{ \pm}(t)-\bar{P}_{ \pm}(t)}{P_{ \pm}(t)+\bar{P}_{ \pm}(t)}= \pm \frac{1}{F_{ \pm}(t)} \sin \left(\Delta M_{s} t\right) \sin \phi_{s}
$$

On the other hand, untagged data samples are sufficient to determine

$$
P_{ \pm}(t)+\bar{P}_{ \pm}(t) \propto\left[\left(1 \pm \cos \phi_{s}\right) e^{-\Gamma_{\mathrm{L}}^{(s)} t}+\left(1 \mp \cos \phi_{s}\right) e^{-\Gamma_{\mathrm{H}}^{(s)} t}\right] .
$$

New-physics effects would be indicated by the following features:

- Sizeable values of the $C P$-violating asymmetries (134).

- The untagged observables (135) depend on two exponentials.

In contrast to (129), these observables do not involve the hadronic parameter $D$ and allow a clean determination of $\phi_{s}$. A detailed discussion of other strategies to search for new physics with $B_{s}$ decays is given in Ref. [74].

\section{The Phenomenology of $B \rightarrow \pi K$ Decays}

In order to probe $\gamma, B \rightarrow \pi K$ decays are very promising. There are already data on these modes available, which triggered a lot of theoretical work. From 1997 until 2000, CLEO reported results on CP-averaged branching ratios $\langle\mathrm{BR}\rangle$; in 1999, also studies of $C P$ asymmetries were reported [91]. In the summer of 2000, BaBar and Belle announced their first $B \rightarrow \pi K$ branching ratios $[92,93]$. These results are collected in Table 1. 

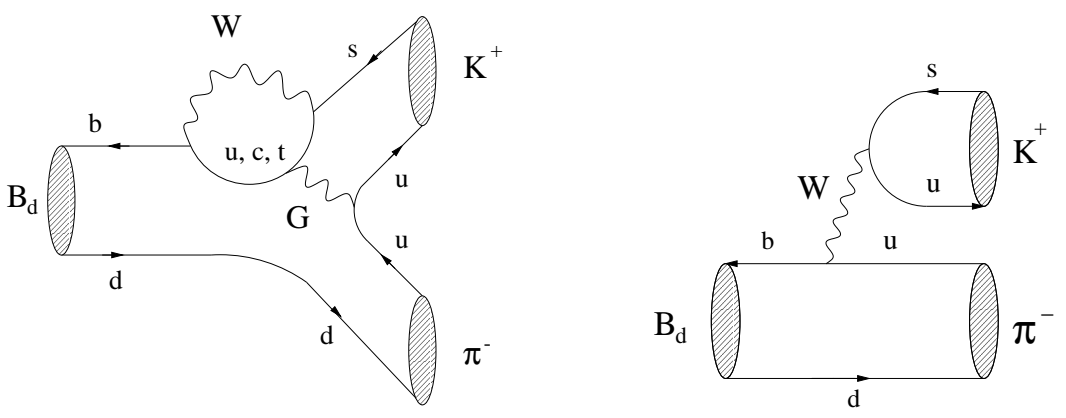

Figure 19. Feynman diagrams contributing to $B_{d}^{0} \rightarrow \pi^{-} K^{+}$.

\subsection{GENERAL REMARKS}

To get more familiar with $B \rightarrow \pi K$ modes, let us consider $B_{d}^{0} \rightarrow \pi^{-} K^{+}$. This channel receives contributions from penguin and colour-allowed treediagram-like topologies, as can be seen in Fig. 19. Because of the small ratio $\left|V_{u s} V_{u b}^{*} /\left(V_{t s} V_{t b}^{*}\right)\right| \approx 0.02$, the QCD penguin topologies dominate this decay, despite their loop suppression. This interesting feature applies to all $B \rightarrow \pi K$ modes. Because of the large top-quark mass, we have also to care about EW penguins. However, in the case of $B_{d}^{0} \rightarrow \pi^{-} K^{+}$and $B^{+} \rightarrow$ $\pi^{+} K^{0}$, these topologies contribute only in colour-suppressed form and are hence expected to play a minor role. On the other hand, EW penguins contribute also in colour-allowed form to $B^{+} \rightarrow \pi^{0} K^{+}$and $B_{d}^{0} \rightarrow \pi^{0} K^{0}$, and may here even compete with tree-diagram-like topologies. Because of the penguin dominance, $B \rightarrow \pi K$ modes represent sensitive probes for new-physics effects [94, 95].

In the Standard Model, the $S U(2)$ isospin symmetry implies

$$
\begin{aligned}
& \sqrt{2} A\left(B^{+} \rightarrow \pi^{0} K^{+}\right)+A\left(B^{+} \rightarrow \pi^{+} K^{0}\right) \\
= & \sqrt{2} A\left(B_{d}^{0} \rightarrow \pi^{0} K^{0}\right)+A\left(B_{d}^{0} \rightarrow \pi^{-} K^{+}\right) \\
= & -\left[(T+C)+\left(P_{\mathrm{ew}}+P_{\mathrm{ew}}^{\mathrm{C}}\right)\right] \propto\left[e^{i \gamma}+q_{\mathrm{ew}}\right],
\end{aligned}
$$

where the $(T+C)$ and $\left(P_{\mathrm{ew}}+P_{\mathrm{ew}}^{\mathrm{C}}\right)$ amplitudes are due to (colour-allowed + colour-suppressed) tree-diagram-like and EW penguin topologies, respectively. A relation with an analogous phase structure holds also for the "mixed" $B^{+} \rightarrow \pi^{+} K^{0}, B_{d}^{0} \rightarrow \pi^{-} K^{+}$system. So far, strategies to probe $\gamma$ have focused on the following systems: $B_{d} \rightarrow \pi^{\mp} K^{ \pm}, B^{ \pm} \rightarrow \pi^{ \pm} K$ ("mixed") [96]-[98], and $B^{ \pm} \rightarrow \pi^{0} K^{ \pm}, B^{ \pm} \rightarrow \pi^{ \pm} K$ ("charged") [99]. Recently, it was pointed out that also the neutral combination $B_{d} \rightarrow \pi^{\mp} K^{ \pm}, B_{d} \rightarrow \pi^{0} K$ is very promising $[50,100]$. 
Interestingly, already $C P$-averaged $B \rightarrow \pi K$ branching ratios may lead to non-trivial constraints on $\gamma$, which rely on flavour-symmetry arguments, involving either $S U(2)$ or $S U(3)$ [101, 102], and dynamical assumptions, concerning mainly the smallness of certain rescattering effects [103]. An example is $B^{+} \rightarrow\left\{\pi^{0} K^{+}, \pi^{0} K^{*+}, \ldots\right\} \rightarrow \pi^{+} K^{0}$. There is still no theoretical consensus on the importance of such final-state interaction (FSI) processes, although, for instance, the "QCD factorization" approach [34] does not suggest large effects. However, there are also experimental indicators for large FSI effects, for example the decays $B^{+} \rightarrow K^{+} \overline{K^{0}}$ and $B_{d} \rightarrow K^{+} K^{-}$, and methods to include them in the strategies to probe $\gamma[98,104]$. So far, these decays have not been observed and the rather strong experimental upper bounds on their $C P$-averaged branching ratios are not in favour of dramatic FSI effects [100], as advocated in some of the papers given in Ref. [103]. Let us therefore neglect these effects for a moment.

\subsection{A SIMPLE EXAMPLE: THE "MIXED" $B \rightarrow \pi K$ SYSTEM}

Let us illustrate the derivation of the constraints on $\gamma$ by considering the "mixed" $B^{+} \rightarrow \pi^{+} K^{0}, B_{d}^{0} \rightarrow \pi^{-} K^{+}$system [97, 98]. If we neglect annihilation topologies, penguins with internal up quarks and colour-suppressed EW penguins, which are expected to play a very minor role (but may be enhanced by large FSI effects; see Subsection 9.1), we may write

$$
\begin{aligned}
A\left(B^{+} \rightarrow \pi^{+} K^{0}\right) & =\tilde{P} \equiv-|\tilde{P}| e^{i \delta_{\tilde{P}}} \\
A\left(B_{d}^{0} \rightarrow \pi^{-} K^{+}\right) & =-[P+T] \equiv-\left[-|P| e^{i \delta_{P}}+|T| e^{i \delta_{T}} e^{i \gamma}\right] .
\end{aligned}
$$

Here $P$ and $T$ describe the penguin and tree-diagram-like topologies shown in Fig. 19, and $\delta_{\tilde{P}}$ and $\delta_{P}$ are $C P$-conserving strong phases. It is convenient to re-write the $B_{d}^{0} \rightarrow \pi^{-} K^{+}$decay amplitude as

$$
A\left(B_{d}^{0} \rightarrow \pi^{-} K^{+}\right)=|P| e^{i \delta_{P}}\left[1-r e^{i \delta} e^{i \gamma}\right],
$$

where $r \equiv|T| /|P|$ and $\delta \equiv \delta_{T}-\delta_{P}$. Consequently, we obtain the following expressions for the $C P$-averaged decay amplitudes:

$$
\begin{aligned}
\left\langle\left|A\left(B^{ \pm} \rightarrow \pi^{ \pm} K\right)\right|^{2}\right\rangle & =|\tilde{P}|^{2} \\
\left\langle\left|A\left(B_{d} \rightarrow \pi^{\mp} K^{ \pm}\right)\right|^{2}\right\rangle & =|P|^{2}\left[1-2 r \cos \delta \cos \gamma+r^{2}\right] .
\end{aligned}
$$

Since the $S U(2)$ isospin symmetry implies $|\tilde{P}|=|P|,{ }^{4}$ we arrive at

$$
R \equiv \frac{\mathrm{BR}\left(B_{d} \rightarrow \pi^{\mp} K^{ \pm}\right)}{\mathrm{BR}\left(B^{ \pm} \rightarrow \pi^{ \pm} K\right)}=1-2 r \cos \delta \cos \gamma+r^{2} .
$$

\footnotetext{
${ }^{4}$ Moreover, $A\left(B^{+} \rightarrow \pi^{+} K^{0}\right)+A\left(B_{d}^{0} \rightarrow \pi^{-} K^{+}\right)=-T$, which corresponds to (136).
} 
Keeping now the hadronic quantities $\delta$ and $r$ as "free" parameters yields

$$
R \geq \sin ^{2} \gamma,
$$

which implies $0^{\circ} \leq \gamma \leq \gamma_{0} \vee 180^{\circ}-\gamma_{0} \leq \gamma \leq 180^{\circ}$ [97], with

$$
\gamma_{0}=\arccos (\sqrt{1-R}) .
$$

This constraint on $\gamma$ is only effective if $R$ is found to be smaller than one. In 1997, when CLEO reported the first result on the $C P$-averaged $B^{+} \rightarrow \pi^{+} K^{0}, B_{d}^{0} \rightarrow \pi^{-} K^{+}$branching ratios, the result was $R=0.65 \pm 0.40$. The central value $R=0.65$ would imply $\gamma_{0}=54^{\circ}$, thereby excluding a large range in the $\bar{\rho}-\bar{\eta}$ plane. Using the present CLEO data [91], we obtain $R=0.95 \pm 0.28$. Unfortunately, the present experimental uncertainties are too large to draw any conclusions and to decide whether $R<1$. If the parameter $r$ is fixed, for example through "factorization" [96], stronger constraints on $\gamma$ can be obtained, which are also effective for $R>1$ [50,51].

\subsection{THE GENERAL $B \rightarrow \pi K$ CASE}

In order to constrain $\gamma$, the key quantities are the following ratios:

$$
\begin{aligned}
R & \equiv \frac{\mathrm{BR}\left(B_{d} \rightarrow \pi^{\mp} K^{ \pm}\right)}{\mathrm{BR}\left(B^{ \pm} \rightarrow \pi^{ \pm} K\right)}=0.95 \pm 0.28 \\
R_{\mathrm{c}} & \equiv \frac{2 \mathrm{BR}\left(B^{ \pm} \rightarrow \pi^{0} K^{ \pm}\right)}{\mathrm{BR}\left(B^{ \pm} \rightarrow \pi^{ \pm} K\right)}=1.27 \pm 0.47 \\
R_{\mathrm{n}} & \equiv \frac{\operatorname{BR}\left(B_{d} \rightarrow \pi^{\mp} K^{ \pm}\right)}{2 \mathrm{BR}\left(B_{d} \rightarrow \pi^{0} K\right)}=0.59 \pm 0.27
\end{aligned}
$$

where we have also taken into account the CLEO results reported in [91]. If we employ the $S U(2)$ flavour symmetry and certain dynamical assumptions, concerning mainly the smallness of FSI effects, we may derive a general parametrization for (145)-(147) (for an explicit example, see (142)) [50],

$$
R_{(\mathrm{c}, \mathrm{n})}=R_{(\mathrm{c}, \mathrm{n})}\left(\gamma, q_{(\mathrm{c}, \mathrm{n})}, r_{(\mathrm{c}, \mathrm{n})}, \delta_{(\mathrm{c}, \mathrm{n})}\right),
$$

where $q_{(\mathrm{c}, \mathrm{n})}$ denotes the ratio of EW penguins to "trees", $r_{(\mathrm{c}, \mathrm{n})}$ is the ratio of "trees" to QCD penguins, and $\delta_{(\mathrm{c}, \mathrm{n})}$ is the $C P$-conserving strong phase between "tree" and QCD penguin amplitudes.

The $q_{(\mathrm{c}, \mathrm{n})}$ can be fixed through theoretical arguments: in the "mixed" $B \rightarrow \pi K$ system, we have $q \approx 0$, as EW penguins contribute only in coloursuppressed form; in the charged [99] and neutral [50] $B \rightarrow \pi K$ systems, $q_{\mathrm{c}}$ and $q_{\mathrm{n}}$ can be fixed through the $S U(3)$ flavour symmetry. On the other hand, the $r_{(\mathrm{c}, \mathrm{n})}$ can be determined with the help of additional experimental 
information: in the "mixed" system, $r$ can be fixed, for example, through arguments based on "factorization" [96], whereas $r_{\mathrm{c}}$ and $r_{\mathrm{n}}$ can be determined from $B^{+} \rightarrow \pi^{+} \pi^{0}$ by using again the $S U(3)$ flavour symmetry [101].

At this point, a comment on the FSI effects discussed in Subsection 9.1 is in order. Whereas the determination of $q$ and $r$ as sketched above may be affected by FSI effects, this is not the case for $q_{\mathrm{c}, \mathrm{n}}$ and $r_{\mathrm{c}, \mathrm{n}}$, since here $S U(3)$ suffices. Nevertheless, we have to assume that $B^{+} \rightarrow \pi^{+} K^{0}$ or $B_{d}^{0} \rightarrow \pi^{0} K^{0}$ do not involve a $C P$-violating weak phase, i.e.

$$
A\left(B^{+} \rightarrow \pi^{+} K^{0}\right)=-|\tilde{P}| e^{i \delta_{\tilde{P}}}=A\left(B^{-} \rightarrow \pi^{-} \overline{K^{0}}\right) .
$$

This relation may be affected by rescattering processes as follows:

$$
A\left(B^{+} \rightarrow \pi^{+} K^{0}\right)=-|\tilde{P}| e^{i \delta_{\tilde{P}}}\left[1+\rho_{\mathrm{c}} e^{i \theta_{\mathrm{c}}} e^{i \gamma}\right],
$$

where $\rho_{\mathrm{c}}$ is doubly Cabibbo-suppressed and is naively expected to be negligibly small. In the "QCD factorization" approach [34], there is no significant enhancement of $\rho_{\mathrm{c}}$ through rescattering processes. However, as we have already noted, there is still no theoretical consensus on the importance of FSI effects. In the charged $B \rightarrow \pi K$ strategy to probe $\gamma$, they can be taken into account through $S U(3)$ flavour-symmetry arguments and additional data on $B^{ \pm} \rightarrow K^{ \pm} K$ decays. On the other hand, in the case of the neutral strategy, FSI effects can be included in an exact manner with the help of the mixing-induced $C P$ asymmetry $\mathcal{A}_{\mathrm{CP}}^{\mathrm{mix}}\left(B_{d} \rightarrow \pi^{0} K_{\mathrm{S}}\right)$ [50].

In contrast to $q_{(\mathrm{c}, \mathrm{n})}$ and $r_{(\mathrm{c}, \mathrm{n})}$, the strong phase $\delta_{(\mathrm{c}, \mathrm{n})}$ suffers from large hadronic uncertainties and is essentially unknown. However, we can get rid of $\delta_{(\mathrm{c}, \mathrm{n})}$ by keeping it as a "free" variable, yielding minimal and maximal values for $R_{(\mathrm{c}, \mathrm{n})}$ :

$$
\left.R_{(\mathrm{c}, \mathrm{n})}^{\mathrm{ext}}\right|_{\delta_{(\mathrm{c}, \mathrm{n})}}=\operatorname{function}\left(\gamma, q_{(\mathrm{c}, \mathrm{n})}, r_{(\mathrm{c}, \mathrm{n})}\right)
$$

Keeping in addition $r_{(\mathrm{c}, \mathrm{n})}$ as a free variable, we obtain another - less restrictive - minimal value for $R_{(\mathrm{c}, \mathrm{n})}$ :

$$
\left.R_{(\mathrm{c}, \mathrm{n})}^{\min }\right|_{r_{(\mathrm{c}, \mathrm{n})}, \delta_{(\mathrm{c}, \mathrm{n})}}=\kappa\left(\gamma, q_{(\mathrm{c}, \mathrm{n})}\right) \sin ^{2} \gamma
$$

In Fig. 20, we show the dependence of (151) and (152) on $\gamma$ for the neutral $B \rightarrow \pi K$ system. ${ }^{5}$ Here the crossed region below the $R_{\text {min }}$ curve, which is described by (152), is excluded. On the other hand, the shaded region is the allowed range (151) for $R_{\mathrm{n}}$, arising in the case of $r_{\mathrm{n}}=0.17$. Fig. 20 allows

\footnotetext{
${ }^{5}$ The charged $B \rightarrow \pi K$ curves look very similar.
} 


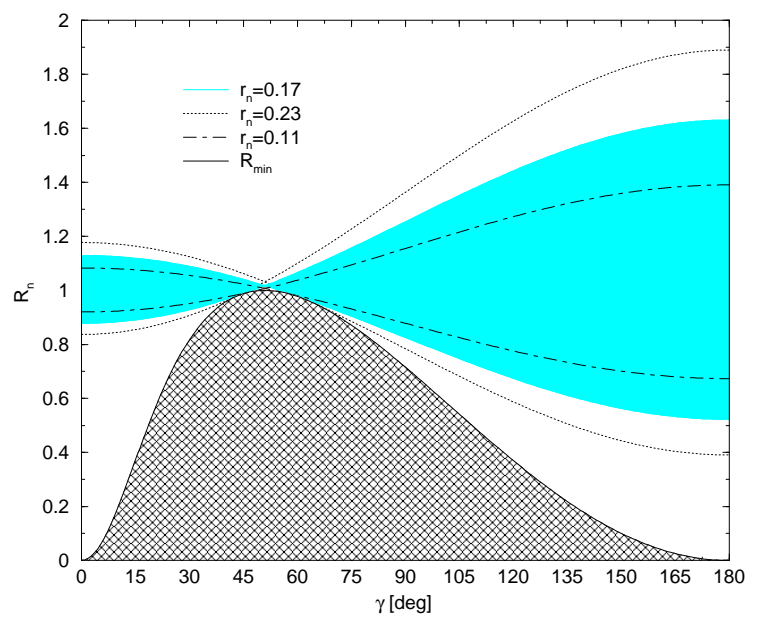

Figure 20. The dependence of the extremal values of $R_{\mathrm{n}}$ on $\gamma$ for $q_{\mathrm{n}}=0.63$.

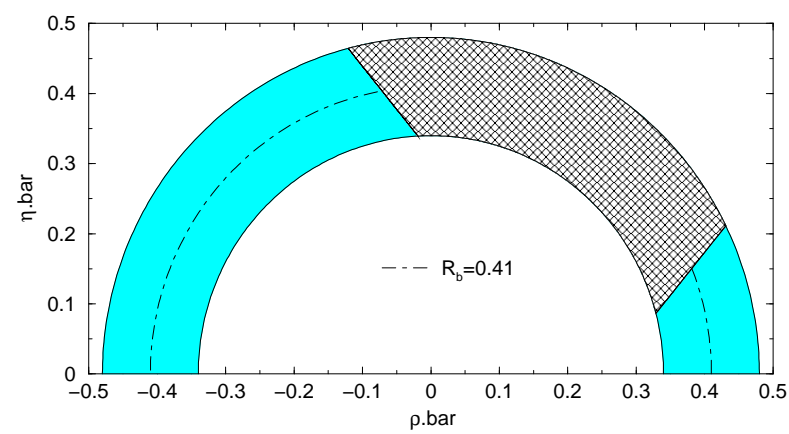

Figure 21. The constraints in the $\bar{\varrho}-\bar{\eta}$ plane for $R_{\mathrm{n}}=0.6$ and $q_{\mathrm{n}}=0.63 \times\left[0.41 / R_{b}\right]$.

us to read off immediately the allowed region for $\gamma$ for a given value of $R_{\mathrm{n}}$. Using the central value of the present CLEO result (147), $R_{\mathrm{n}}=0.6$, the $R_{\text {min }}$ curve implies $0^{\circ} \leq \gamma \leq 21^{\circ} \vee 100^{\circ} \leq \gamma \leq 180^{\circ}$. The corresponding situation in the $\bar{\varrho}-\bar{\eta}$ plane is shown in Fig. 21, where the crossed region is excluded and the circles correspond to $R_{b}=0.41 \pm 0.07$. As the theoretical expression for $q_{\mathrm{n}}$ is proportional to $1 / R_{b}$, the constraints in the $\bar{\varrho}-\bar{\eta}$ plane are actually more appropriate than the constraints on $\gamma$.

If we use additional information on $r_{\mathrm{n}}$, we may put even stronger constraints on $\gamma$. For $r_{\mathrm{n}}=0.17$, we obtain the allowed range $138^{\circ} \leq \gamma \leq 180^{\circ}$. It is interesting to note that the $R_{\min }$ curve is only effective for $R_{\mathrm{n}}<1$, which is favoured by the most recent CLEO data [91]. A similar pattern is also exhibited by the first Belle results [93], yielding $R_{\mathrm{n}}=0.4 \pm 0.2$.

For the central value $R_{\mathrm{c}}=1.3,(152)$ is not effective and $r_{\mathrm{c}}$ has to be fixed to constrain $\gamma$. Using $r_{\mathrm{c}}=0.21$, we obtain $87^{\circ} \leq \gamma \leq 180^{\circ}$. Although 
it is too early to draw definite conclusions, it should be emphasized that the present CLEO results on $R_{(\mathrm{c}, \mathrm{n})}$ prefer the second quadrant for $\gamma$, i.e. $\gamma \geq 90^{\circ}$. Similar conclusions were also obtained using other $B \rightarrow \pi K, \pi \pi$ strategies [105]. Interestingly, such a situation would be in conflict with the standard analysis of the unitarity triangle [21], yielding $38^{\circ} \leq \gamma \leq 81^{\circ}$.

The $R_{(\mathrm{c}, \mathrm{n})}$ allow us to determine $\cos \delta_{(\mathrm{c}, \mathrm{n})}$ as functions of $\gamma$, thereby providing also constraints on the strong phases $\delta_{(\mathrm{c}, \mathrm{n})}$ [100]. Interestingly, the present CLEO data are in favour of $\cos \delta_{\mathrm{n}}<0$, which would be in conflict with "factorization". Moreover, they point towards a positive value of $\cos \delta_{\mathrm{c}}$, which would be in conflict with the theoretical expectation of equal signs for $\cos \delta_{\mathrm{c}}$ and $\cos \delta_{\mathrm{n}}$.

If future data should confirm the "puzzling" situation for $\gamma$ and $\cos \delta_{\mathrm{c}, \mathrm{n}}$, which is favoured by the present $B \rightarrow \pi K$ CLEO data, it may be an indication for new-physics contributions to the EW penguin sector, or a manifestation of large flavour-symmetry-breaking effects. In order to distinguish between these possibilities, further studies are needed. As soon as $C P$ asymmetries in $B_{d} \rightarrow \pi^{\mp} K^{ \pm}$or $B^{ \pm} \rightarrow \pi^{0} K^{ \pm}$are observed,

$$
A_{\mathrm{CP}}^{(\mathrm{c}, \mathrm{n})}=A_{\mathrm{CP}}^{(\mathrm{c}, \mathrm{n})}\left(\gamma, q_{(\mathrm{c}, \mathrm{n})}, r_{(\mathrm{c}, \mathrm{n})}, \delta_{(\mathrm{c}, \mathrm{n})}\right),
$$

we may even determine $\gamma$ and $\delta_{(\mathrm{c}, \mathrm{n})}$. Here we may also arrive at a situation, where the $B \rightarrow \pi K$ observables do not provide any solution for these quantities, which would be an immediate indication for new physics [95].

\subsection{TOWARDS THE CALCULATION OF $B \rightarrow \pi K, \pi \pi$}

Calculations of $B \rightarrow \pi K, \pi \pi$ modes are usually based on a perturbative picture, where strong phases are obtained from absorptive parts of loop diagrams. This approach is referred to as the "Bander-Silverman-Soni (BSS) mechanism" [106]. Recently, a conceptual improvement of this formalism was presented in Ref. [34] (see also [107]), where it is argued that there is a heavy-quark expansion for non-leptonic $B$-decays into two light mesons, yielding transition amplitudes of the structure given in (41). In this approach, soft non-factorizable contributions and FSI effects are suppressed by $\Lambda_{\mathrm{QCD}} / m_{b}$. However, the question arises whether the $b$-quark mass is large enough to suppress these terms sufficiently. Moreover, there are problems due to "chirally enhanced" terms, which are formally suppressed by $1 / m_{b}$, but are numerically of $\mathcal{O}(1)$. Another "perturbative" QCD approach to deal with non-leptonic charmless $B$ decays was developed in Ref. [108].

Many calculations of $B \rightarrow \pi K, \pi \pi$ can be found in the literature (see, for instance, [109]). The results for the $C P$-averaged branching ratios are generally in rather good agreement with the CLEO data. However, there are two exceptions: $B_{d} \rightarrow \pi^{+} \pi^{-}$and $B_{d} \rightarrow \pi^{0} K$. Concerning the former decay, 
the calculations favour a value at the $7 \times 10^{-6}$ level, whereas CLEO finds $\mathrm{BR}\left(B_{d} \rightarrow \pi^{+} \pi^{-}\right)=\left(4.3_{-1.4}^{+1.6} \pm 0.5\right) \times 10^{-6}$. Due to interference between treediagram-like and penguin topologies, the theoretical predictions depend on $\gamma$; the CLEO result would be in favour of $\gamma>90^{\circ}$ [105]. On the other hand, the first $\mathrm{BaBar}$ result is $\operatorname{BR}\left(B_{d} \rightarrow \pi^{+} \pi^{-}\right)=\left(9.3_{-2.3-1.4}^{+2.6+1.2}\right) \times 10^{-6}$. Concerning $B_{d} \rightarrow \pi^{0} K$, the calculations favour a value at the $5 \times 10^{-6}$ level, which is essentially independent of $\gamma$ and smaller than the CLEO result $\operatorname{BR}\left(B_{d} \rightarrow \pi^{0} K\right)=\left(14.6_{-5.1-3.3}^{+5.9+2.4}\right) \times 10^{-6}$. Interestingly, the first Belle result $\left(21_{-7.8-2.3}^{+9.3+2.5}\right) \times 10^{-6}$ is also in favour of a large $C P$-averaged $B_{d} \rightarrow \pi^{0} K$ branching ratio. Because of the large experimental uncertainties, we cannot yet draw any definite conclusions. However, the experimental situation should improve significantly in the future.

\section{The $B_{d} \rightarrow \pi^{+} \pi^{-}, B_{s} \rightarrow K^{+} K^{-}$System}

As we have seen in Subsection $6.2, B_{d} \rightarrow \pi^{+} \pi^{-}$is usually considered as a tool to determine $\alpha=180^{\circ}-\beta-\gamma$. Unfortunately, the extraction of $\alpha$ from $\mathcal{A}_{\mathrm{CP}}^{\mathrm{mix}}\left(B_{d} \rightarrow \pi^{+} \pi^{-}\right)$is affected by large penguin uncertainties, and the strategies to control them through additional data are challenging. In this section, we discuss a new approach to employ $B_{d} \rightarrow \pi^{+} \pi^{-}$: combining this mode with $B_{s} \rightarrow K^{+} K^{-}$- its $U$-spin ${ }^{6}$ counterpart - a simultaneous determination of $\phi_{d}=2 \beta$ and $\gamma$ becomes possible [45]. In this method, no model-dependent assumptions are required, and FSI effects, which led to considerable attention in the $B \rightarrow \pi K$ strategies to probe $\gamma$, do not lead to any problems. The theoretical accuracy is hence only limited by $U$-spinbreaking effects. This approach is promising for CDF-II $\left(\left.\Delta \gamma\right|_{\exp }=\mathcal{O}\left(10^{\circ}\right)\right)$ [59], and ideally suited for $\operatorname{LHCb}\left(\left.\Delta \gamma\right|_{\text {exp }}=\mathcal{O}\left(1^{\circ}\right)\right)$ [44].

There are also other interesting strategies to determine $\gamma$ with the help of the $U$-spin symmetry: $B_{s(d)} \rightarrow J / \psi K_{\mathrm{S}}, B_{d(s)} \rightarrow D_{d(s)}^{+} D_{d(s)}^{-}[36]$ or $B_{s(d)} \rightarrow J / \psi \eta[110]$, as well as $B_{(s)} \rightarrow \pi K$ [111]. For the "prehistory" of $U$-spin arguments in $B$ decays, the reader is referred to Ref. [112].

\subsection{EXTRACTION OF $\beta$ AND $\gamma$}

Looking at the diagrams shown in Fig. 11, we observe that $B_{s} \rightarrow K^{+} K^{-}$is obtained from $B_{d} \rightarrow \pi^{+} \pi^{-}$by interchanging all down and strange quarks. The structure of the corresponding decay amplitudes is given as follows:

$$
A\left(B_{d}^{0} \rightarrow \pi^{+} \pi^{-}\right)=\left(1-\frac{\lambda^{2}}{2}\right) \mathcal{C}\left[e^{i \gamma}-d e^{i \theta}\right]
$$

\footnotetext{
${ }^{6} U$-spin is a subgroup of $S U(3)_{\mathrm{F}}$, relating down and strange quarks to each other.
} 


$$
A\left(B_{s}^{0} \rightarrow K^{+} K^{-}\right)=\lambda \mathcal{C}^{\prime}\left[e^{i \gamma}+\left(\frac{1-\lambda^{2}}{\lambda^{2}}\right) d^{\prime} e^{i \theta^{\prime}}\right]
$$

where $\mathcal{C}^{\prime}$ and $d^{\prime} e^{i \theta^{\prime}}$ take the same form as $\mathcal{C}$ and $d e^{i \theta}$ (see (93) and (94)). Using the formalism discussed in Subsection 5.3 yields

$$
\begin{aligned}
\mathcal{A}_{\mathrm{CP}}^{\mathrm{dir}}\left(B_{d} \rightarrow \pi^{+} \pi^{-}\right) & =\text {function }(d, \theta, \gamma) \\
\mathcal{A}_{\mathrm{CP}}^{\mathrm{mix}}\left(B_{d} \rightarrow \pi^{+} \pi^{-}\right) & =\text {function }\left(d, \theta, \gamma, \phi_{d}=2 \beta\right) \\
\mathcal{A}_{\mathrm{CP}}^{\mathrm{dir}}\left(B_{s} \rightarrow K^{+} K^{-}\right) & =\text {function }\left(d^{\prime}, \theta^{\prime}, \gamma\right) \\
\mathcal{A}_{\mathrm{CP}}^{\mathrm{mix}}\left(B_{s} \rightarrow K^{+} K^{-}\right) & =\text {function }\left(d^{\prime}, \theta^{\prime}, \gamma, \phi_{s} \approx 0\right),
\end{aligned}
$$

where the Standard-Model expectation $\phi_{s} \approx 0$ can be probed through the decay $B_{s} \rightarrow J / \psi \phi$. Consequently, we have four observables, depending on six "unknowns". However, since $B_{d} \rightarrow \pi^{+} \pi^{-}$and $B_{s} \rightarrow K^{+} K^{-}$are related to each other by interchanging all down and strange quarks, the $U$-spin flavour symmetry of strong interactions implies

$$
d^{\prime} e^{i \theta^{\prime}}=d e^{i \theta}
$$

Using this relation, the four observables (156)-(159) depend on the four quantities $d, \theta, \phi_{d}=2 \beta$ and $\gamma$, which can hence be determined.

\subsection{MINIMAL USE OF THE U-SPIN SYMMETRY}

The $U$-spin arguments can be "minimized", if we employ $\phi_{d}=2 \beta$ as an input, which can be determined straightforwardly from $B_{d} \rightarrow J / \psi K_{\mathrm{S}}$. The observables $\mathcal{A}_{\mathrm{CP}}^{\text {dir }}\left(B_{d} \rightarrow \pi^{+} \pi^{-}\right)$and $\mathcal{A}_{\mathrm{CP}}^{\text {mix }}\left(B_{d} \rightarrow \pi^{+} \pi^{-}\right)$allow us then to eliminate the strong phase $\theta$ and to determine $d$ as a function of $\gamma$. Analogously, $\mathcal{A}_{\mathrm{CP}}^{\mathrm{dir}}\left(B_{s} \rightarrow K^{+} K^{-}\right)$and $\mathcal{A}_{\mathrm{CP}}^{\mathrm{mix}}\left(B_{s} \rightarrow K^{+} K^{-}\right)$allow us to eliminate the strong phase $\theta^{\prime}$ and to determine $d^{\prime}$ as a function of $\gamma$. The corresponding contours in the $\gamma-d$ and $\gamma-d^{\prime}$ planes can be fixed in a theoretically clean way. Using the $U$-spin relation $d^{\prime}=d$, these contours allow the determination both of the CKM angle $\gamma$ and of the hadronic quantities $d, \theta, \theta^{\prime}$.

Let us illustrate this approach by considering the following example:

$$
\begin{array}{llll}
\mathcal{A}_{\mathrm{CP}}^{\mathrm{dir}}\left(B_{d} \rightarrow \pi^{+} \pi^{-}\right) & =+24 \%, & \mathcal{A}_{\mathrm{CP}}^{\operatorname{mix}}\left(B_{d} \rightarrow \pi^{+} \pi^{-}\right) & =+4.4 \%, \\
\mathcal{A}_{\mathrm{CP}}^{\mathrm{dir}}\left(B_{s} \rightarrow K^{+} K^{-}\right) & =-17 \%, & \mathcal{A}_{\mathrm{CP}}^{\mathrm{mix}}\left(B_{s} \rightarrow K^{+} K^{-}\right) & =-28 \%,
\end{array}
$$

which corresponds to the input parameters $d=d^{\prime}=0.3, \theta=\theta^{\prime}=210^{\circ}$, $\phi_{s}=0, \phi_{d}=53^{\circ}$ and $\gamma=76^{\circ}$. In Fig. 22, the corresponding contours in the $\gamma-d$ and $\gamma-d^{\prime}$ planes are represented by the solid and dot-dashed lines, 


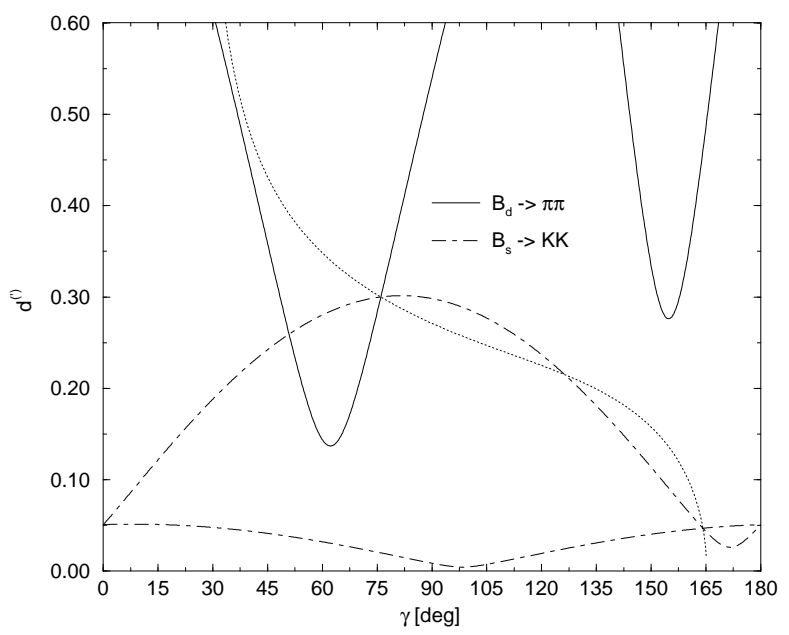

Figure 22. The contours in the $\gamma-d^{\left({ }^{\prime}\right)}$ planes fixed through the $C P$-violating $B_{d} \rightarrow \pi^{+} \pi^{-}$ and $B_{s} \rightarrow K^{+} K^{-}$observables for an example discussed in the text.

respectively. Their intersection yields a twofold solution for $\gamma$, given by $51^{\circ}$ and our input value of $76^{\circ}$. The dotted line is related to the quantity

$$
K \equiv-\left(\frac{1-\lambda^{2}}{\lambda^{2}}\right)\left[\frac{\mathcal{A}_{\mathrm{CP}}^{\mathrm{dir}}\left(B_{d} \rightarrow \pi^{+} \pi^{-}\right)}{\mathcal{A}_{\mathrm{CP}}^{\mathrm{dir}}\left(B_{s} \rightarrow K^{+} K^{-}\right)}\right],
$$

which can be combined with $\mathcal{A}_{\mathrm{CP}}^{\operatorname{mix}}\left(B_{s} \rightarrow K^{+} K^{-}\right)$through the $U$-spin relation (160) to fix another contour in the $\gamma-d$ plane. Combining all contours in Fig. 22 with one another, we obtain a single solution for $\gamma$, which is given by the "true" value of $76^{\circ}$. As an interesting by-product, the penguin parameters $d$ and $\theta, \theta^{\prime}$ can be determined as well, allowing a comparison with theoretical predictions and valuable insights into hadronic physics.

\subsection{U-SPIN-BREAKING CORRECTIONS}

It should be emphasized that the theoretical accuracy of $\gamma$ and the hadronic parameters $d, \theta, \theta^{\prime}$ is only limited by $U$-spin-breaking effects. In particular, it is not affected by FSI or penguin effects. Interestingly, the relation (160) does not receive $U$-spin-breaking corrections within a modernized version of the "Bander-Silverman-Soni mechanism" [106], making use - among other things - of the factorization hypothesis to estimate the relevant hadronic matrix elements [45]. This remarkable feature strengthens our confidence into the $U$-spin relations used for the extraction of $\beta$ and $\gamma$. However, further theoretical studies of the $U$-spin-breaking effects in the $B_{d} \rightarrow \pi^{+} \pi^{-}$, $B_{s} \rightarrow K^{+} K^{-}$system, employing, for example, the "QCD factorization" approach [34], would be desirable. 
Apart from these theoretical considerations, it is also possible to obtain experimental insights into $U$-spin breaking. A first consistency check is provided by $\theta=\theta^{\prime}$. Moreover, we may determine the normalization factors $|\mathcal{C}|$ and $\left|\mathcal{C}^{\prime}\right|$ of the $B_{d}^{0} \rightarrow \pi^{+} \pi^{-}$and $B_{s}^{0} \rightarrow K^{+} K^{-}$decay amplitudes (see (154) and (155)) with the help of the corresponding $C P$-averaged branching ratios. Comparing them with the "factorized" result

$$
\left|\frac{\mathcal{C}^{\prime}}{\mathcal{C}}\right|_{\text {fact }}=\left[\frac{f_{K}}{f_{\pi}}\right]\left[\frac{F_{B_{s} K}\left(M_{K}^{2} ; 0^{+}\right)}{F_{B_{d}}\left(M_{\pi}^{2} ; 0^{+}\right)}\right]\left(\frac{M_{B_{s}}^{2}-M_{K}^{2}}{M_{B_{d}}^{2}-M_{\pi}^{2}}\right)
$$

we have another interesting probe for $U$-spin-breaking effects. Moreover, the $U$-spin relation (160) also implies

$$
\left[\frac{\mathcal{A}_{\mathrm{CP}}^{\mathrm{dir}}\left(B_{s} \rightarrow K^{+} K^{-}\right)}{\mathcal{A}_{\mathrm{CP}}^{\mathrm{dir}}\left(B_{d} \rightarrow \pi^{+} \pi^{-}\right)}\right]=-\left|\frac{\mathcal{C}^{\prime}}{\mathcal{C}}\right|^{2} \times\left[\frac{\mathrm{BR}\left(B_{d} \rightarrow \pi^{+} \pi^{-}\right)}{\mathrm{BR}\left(B_{s} \rightarrow K^{+} K^{-}\right)}\right] .
$$

In order to obtain further insights, the $B_{d} \rightarrow \rho^{+} \rho^{-}, B_{s} \rightarrow K^{*+} K^{*-}$ system would be of particular interest, allowing us to determine $\gamma$ together with the mixing phases $\phi_{d}$ and $\phi_{s}$, and tests of several $U$-spin relations [84]. Further strategies to explore $U$-spin-breaking effects were recently discussed in Ref. [113].

\subsection{SEARCHING FOR NEW PHYSICS}

Since penguin processes play an important role in the decays $B_{s} \rightarrow K^{+} K^{-}$ and $B_{d} \rightarrow \pi^{+} \pi^{-}$, they - and the strategy to determine $\gamma$, where moreover the unitarity of the CKM matrix is employed - may well be affected by new physics. Interestingly, the Standard Model implies a rather restricted region in the space of the $C P$-violating observables of the $B_{s} \rightarrow K^{+} K^{-}$, $B_{d} \rightarrow \pi^{+} \pi^{-}$system [95]. A future measurement of observables lying significantly outside of this allowed region would be an immediate indication for new physics. On the other hand, if the observables should lie within the region predicted by the Standard Model, we can extract a value for $\gamma$ by following the strategy discussed above. This value for $\gamma$ may well be in conflict with other approaches, which would then also indicate the presence of new physics.

\section{Remarks on Other Rare Decays}

Let us finally make a few remarks on other "rare" $B$ decays, which occur only at the one-loop level in the Standard Model, and involve $\bar{b} \rightarrow \bar{s}$ or $\bar{b} \rightarrow \bar{d}$ flavour-changing neutral-current transitions: $B \rightarrow K^{*} \gamma, B_{s, d} \rightarrow \mu^{+} \mu^{-}$, $B \rightarrow K^{*} \mu^{+} \mu^{-}$, inclusive decays, etc. Within the Standard Model, these 
transitions exhibit small branching ratios at the $10^{-5} \ldots 10^{-10}$ level, do not show sizeable $C P$-violating effects, and depend on $\left|V_{t s}\right|$ and $\left|V_{t d}\right|$. A measurement of these CKM factors from rare decays would be complementary to the one from $B_{s, d}^{0}-\overline{B_{s, d}^{0}}$ mixing. Since they are absent at the tree level in the Standard Model, rare $B$-decays represent interesting probes to search for new physics, and have many other interesting aspects. For detailed discussions, the reader is referred to the reviews listed in Refs. [27, 114].

\section{Conclusions and Outlook}

The phenomenology of non-leptonic decays of $B$-mesons is very rich and has been a field of very active research over the last couple of years. These modes provide a very fertile testing ground for the Standard-Model description of $C P$ violation, and allow the direct determination of the angles of the unitarity triangles. Here the goal is to overconstrain these triangles, which may open a window to the physics beyond the Standard Model. As by-products, some strategies allow also the determination of interesting hadronic parameters and provide valuable insights into hadronic physics. Moreover, there are many other exciting aspects, for instance the physics potential of certain rare $B$-decays. In view of the rich experimental $B$-physics programme of this decade and the strong interaction between theory and experiment, I have no doubt that an exciting future is ahead of us.

\section{Acknowledgements}

I would like to thank the organizers of this NATO Advanced Study Institute for inviting me to this very interesting meeting in such a pleasant environment. Special thanks go to Gustavo Branco, Gui Rebelo and Juca Silva-Marcos for their splendid hospitality.

\section{References}

1. Christenson, J.H., Cronin, J.W., Fitch, V.L. and Turlay, R. (1964), Phys. Rev. Lett. 13, 138.

2. For reviews, see Nir, Y. (1999), IASSNS-HEP-99-96 [hep-ph/9911321];

Rosner, J.L. (2000), EFI-2000-16 [hep-ph/0005258];

Gronau, M. (1999), SLAC-PUB-8221 [hep-ph/9908343];

Fleischer, R. (2000), Nucl. Instrum. Meth. A446, 1.

3. Branco, G., Lavoura, L. and Silva, J. (1999), CP Violation, Oxford Science Publications, Clarendon Press, Oxford;

Bigi, I.I. and Sanda, A.I. (2000), CP Violation, Cambridge Monographs on Particle Physics, Nuclear Physics and Cosmology, Cambridge University Press, Cambridge.

4. Buras, A.J. and Fleischer, R. (1997), in Heavy Flavours II, p. 65, eds. Buras, A.J. and Lindner, M., World Scientific, Singapore [hep-ph/9704376]. 
5. For reviews, see Grossman, Y., Nir, Y. and Rattazzi, R. (1997), in Heavy Flavours II, p. 755, eds. Buras, A. and Lindner, M., World Scientific, Singapore [hep-ph/9701231]; Gronau, M. and London, D. (1997), Phys. Rev. D55, 2845;

Nir, Y. and Quinn, H.R. (1992), Annu. Rev. Nucl. Part. Sci. 42, 211;

Fleischer, R. (1997), in the proceedings of the 7th International Symposium on Heavy Flavor Physics, Santa Barbara, California, July 7-11, 1997, ed. Campagnari, C., World Scientific, Singapore, p. 155 [hep-ph/9709291];

Wolfenstein, L. (1998), Phys. Rev. D57, 6857.

6. Akhmedov, E., these proceedings.

7. Dick, K., Freund, M., Lindner, M. and Romanino, A. (1999), Nucl. Phys. B562, 29.

8. Sakharov, A.D. (1967), JETP Lett. 5, 24.

9. Buchmüller, W., these proceedings.

10. Rubakov, V.A. and Shaposhnikov, M.E. (1996), Usp. Fiz. Nauk 166, 493; Phys. Usp. 39, 461;

Riotto, A. and Trodden, M. (1999), Annu. Rev. Nucl. Part. Sci. 49, 35.

11. The BaBar Physics Book, eds. Harrison, P. and Quinn, H. (1998), SLAC report 504; Workshop on $B$ Physics at the Tevatron Run II and Beyond, please have a look at http://www-theory.fnal.gov/people/ligeti/Brun2/;

Report of the b-decay Working Group of the Workshop Standard Model Physics (and More) at the LHC, Ball, P. et al. (2000), CERN-TH/2000-101 [hep-ph/0003238].

12. Glashow, S.L. (1961), Nucl. Phys. 22, 579;

Weinberg, S. (1967), Phys. Rev. Lett. 19, 1264;

Salam, A. (1968), in Elementary Particle Theory, ed. Svartholm, N., Almqvist and Wiksell, Stockholm.

13. Cabibbo, N. (1963), Phys. Rev. Lett. 10, 531.

14. Kobayashi, M. and Maskawa, T. (1973), Progr. Theor. Phys. 49, 652.

15. Fritzsch, H. and Xing, Z.-Z. (1997), Phys. Lett. B413 396.

16. Wolfenstein, L. (1983), Phys. Rev. Lett. 51, 1945.

17. Buras, A.J., Lautenbacher, M.E. and Ostermaier, G. (1994), Phys. Rev. D50, 3433.

18. Jarlskog, C. (1985), Phys. Rev. Lett. 55, 1039; Z. Phys. C29, 491.

19. Aleksan, R., Kayser, B. and London, D. (1994), Phys. Rev. Lett. 73, 18.

20. Chau, L.L. and Keung, W.-Y. (1984), Phys. Rev. Lett. 53, 1802;

Jarlskog, C. and Stora, R. (1988), Phys. Lett. B208, 268.

21. Ali, A. and London, D. (2000), DESY 00-026 [hep-ph/0002167].

22. Wolfenstein, L. (1964), Phys. Rev. Lett. 13, 562.

23. KTeV Collaboration (Alavi-Harati, A. et al.) (1999), Phys. Rev. Lett. 83, 22.

24. NA48 Collaboration (Fanti, V. et al.) (1999), Phys. Lett. B465, 335;

for the update given in (23), see http://na48.web.cern.ch/NA48/.

25. For recent reviews, see Buras, A.J. (1999), TUM-HEP-355-99 [hep-ph/9908395];

Eeg, J.O., these proceedings.

26. Buchalla, G. and Buras, A.J. (1994), Phys. Lett. B333, 221.

27. Buras, A.J. (1998), in the proceedings of the Les Houches 1997 Summer School on Theoretical Physics: Probing the Standard Model of Particle Interactions, Les Houches, France, July 28 - September 5, 1997, eds. Gupta, R., Morel, A., de Rafael, E. and David, F., North-Holland, Amsterdam [hep-ph/9806471].

28. Buchalla, G., Buras, A. and Lautenbacher, M. (1996), Rev. Mod. Phys. 68, 1125.

29. Fleischer, R. (1994), Z. Phys. C62, 81; Phys. Lett. B321, 259 and 332, 419.

30. Fleischer, R. (1997), Int. J. Mod. Phys. A12, 2459.

31. Deshpande, N.G. and He, X.-G. (1995), Phys. Rev. Lett. 74, 26 [E: ibid., p. 4099]; Gronau, M., Hernández, O., London, D. and Rosner, J. (1995), Phys. Rev. D52, 6374.

32. See, for instance, Schwinger, J. (1964), Phys. Rev. Lett. 12, 630;

Farikov, D. and Stech, B. (1978), Nucl. Phys. B133, 315;

Cabibbo, N. and Maiani, L. (1978), Phys. Lett. B73, 418 [E: ibid. B76, 663];

Bjorken, J.D. (1989), Nucl. Phys. (Proc. Suppl.) B11, 325;

Dugan, M. and Grinstein, B. (1991), Phys. Lett. B255, 583; 
Politzer, H.D. and Wise, M.B. (1991), Phys. Lett. B257, 399;

Neubert, M. and Stech, B. (1997), in Heavy Flavours II, p. 294, eds. Buras, A.J. and Lindner, M., World Scientific, Singapore [hep-ph/9705292].

33. Buras, A.J., Gérard, J.-M. and Rückl, R. (1986), Nucl. Phys. B268, 16;

Buras, A.J. and Gérard, J.-M. (1988), Phys. Lett. B203, 272.

34. Beneke, M., Buchalla, G., Neubert, M. and Sachrajda, C.T. (1999), Phys. Rev. Lett. 83, 1914; (2000), Nucl. Phys. B591, 313 and PITHA-00-13 [hep-ph/0007256].

35. Carter, A.B. and Sanda, A.I. (1980), Phys. Rev. Lett. 45, 952; (1981), Phys. Rev. D23, 1567;

Bigi, I.I and Sanda, A.I. (1981), Nucl. Phys. B193, 85

36. Fleischer, R. (1999), Eur. Phys. J. C10, 299.

37. CLEO Collaboration (Bonvicini, G. et al.) (2000), Phys. Rev. Lett. 84, 5940.

38. Hitlin, D., BaBar Collaboration, plenary talk at ICHEP 2000, Osaka, Japan, July 27 - August 2, 2000

39. OPAL Collaboration (Ackerstaff, K. et al.) (1998), Eur. Phys. J. C5, 379.

40. CDF Collaboration (Affolder, T. et al.) (2000), Phys. Rev. D61, 072005.

41. ALEPH Collaboration (Barate, R. et al.) (2000), Phys. Lett. B492, 259.

42. Aihara, H., Belle Collaboration, plenary talk at ICHEP 2000, Osaka, Japan, July 27 - August 2, 2000.

43. Kagan, A.L. and Neubert, M. (2000), Phys. Lett. B492, 115;

Silva, J.P. and Wolfenstein, L. (2000), SLAC-PUB-8548 [hep-ph/0008004];

Eyal, G., Nir, Y. and Perez, G. (2000), J. High Energy Phys. 0008, 028;

Xing, Z.-Z. (2000), hep-ph/0008018;

Buras, A.J. and Buras, R. (2000), TUM-HEP-285-00 [hep-ph/0008273].

44. Report of the b-decay Working Group of the Workshop Standard Model Physics (and More) at the LHC, Ball, P. et al. (2000), CERN-TH/2000-101 [hep-ph/0003238].

45. Fleischer, R. (1999), Phys. Lett. B459, 306.

46. Fleischer, R. (2000), Eur. Phys. J. C16, 87.

47. Gronau, M. (1993), Phys. Lett. B300, 163;

Silva, J.P. and Wolfenstein, L. (1994), Phys. Rev. D49, R1151;

Aleksan, R. et al. (1995), Phys. Lett. B356, 95;

Ward, B.F. (1995), Phys. Rev. D51, 6253;

DeJongh, F. and Sphicas, P. (1996), Phys. Rev. D53, 4930;

Ciuchini, M. et al. (1997), Nucl. Phys. B501, 271;

Marrocchesi, P.S. and Paver, N. (1998), Int. J. Mod. Phys. A13, 251;

Ali, A., Kramer, G. and Lü, C.-D. (1999), Phys. Rev. D59, 014005.

48. Gronau, M. and London, D. (1990), Phys. Rev. Lett. 65, 3381.

49. Buras, A.J. and Fleischer, R. (1996), Phys. Lett. B365, 390.

50. Buras, A.J. and Fleischer, R. (1999), Eur. Phys. J. C11, 93.

51. Gronau, M., Pirjol, D. and Yan, T.-M. (1999), Phys. Rev. D60, 034021.

52. Lipkin, H., Nir, Y., Quinn, H. and Snyder, A. (1991), Phys. Rev. D44, 1454.

53. Snyder, A. and Quinn, H. (1993), Phys. Rev. D48, 2139.

54. Quinn, H. and Silva, J. (2000), Phys. Rev. D62, 054002.

55. Deandrea, A., Gatto, R., Ladisa, M., Nardulli, G. and Santorelli, P. (2000), Phys. Rev. D62, 036001.

56. Buras, A.J. and Fleischer, R. (1995), Phys. Lett. B360, 138.

57. Fleischer, R. and Mannel, T. (1997), Phys. Lett. B397, 269.

58. Charles, J. (1999), Phys. Rev. D59, 054007.

59. F. Würthwein, private communication.

60. Sachs, R.G. (1985), EFI-85-22 (unpublished);

Dunietz, I. and Sachs, R.G. (1988), Phys. Rev. D37, 3186 [E: ibid. D39, 3515];

Dunietz, I. (1998), Phys. Lett. B427, 179.

61. The BaBar Physics Book, eds. Harrison, P. and Quinn, H. (1998), SLAC report 504.

62. Gronau, M. and Wyler, D. (1991), Phys. Lett. B265, 172.

63. Atwood, D., Dunietz, I. and Soni, A. (1997), Phys. Rev. Lett. 78, 3257. 
64. Dunietz, I. (1991), Phys. Lett. B270, 75.

65. Masetti, M. (1992), Phys. Lett. B286, 160.

66. Fleischer, R. and Wyler, D. (2000), Phys. Rev. D62, 057503.

67. LEP $B$-oscillation Working Group, see http://lepbosc.web.cern.ch/LEPBOSC/combined_results/sept_ 2000/.

68. Stocchi, A., talk given at ICHEP 2000, Osaka, Japan, July 27 - August 2, 2000.

69. Buras, A.J. (1996), in the proceedings of ICHEP 1996, Warsaw, Poland, July 2531, 1996, eds. Ajduk, Z. and Wroblewski, A.K., World Scientific, Singapore, p. 243 [hep-ph/9610461].

70. Dunietz, I. (1995), Phys. Rev. D52, 3048.

71. Fleischer, R. and Dunietz, I. (1997), Phys. Rev. D55, 259.

72. Fleischer, R. and Dunietz, I. (1996), Phys. Lett. B387, 361.

73. Fleischer, R. (1998), Phys. Rev. D58, 093001.

74. Dunietz, I., Fleischer, R. and Nierste, U. (2000), in preparation.

75. Beneke, M., Buchalla, G., Greub, C., Lenz, A. and Nierste, U. (1999), Phys. Lett. B459, 631.

76. Hashimoto, S., Ishikawa, K., Onogi, T., Sakamoto, M. Tsutsui, N. and Yamada, N. (2000), Phys. Rev. D62, 114502.

77. Becirevic, D., Meloni, D., Retico, A., Gimenez, V., Lubicz, V. and Martinelli, G. (2000), CPHT-RR-057-0600 [hep-ph/0006135].

78. Aleksan, R., Dunietz, I. and Kayser, B. (1992), Z. Phys. C54, 653.

79. Gronau, M. and London, D. (1991), Phys. Lett. B253, 483.

80. London, D., Sinha, N. and Sinha, R. (2000), Phys. Rev. Lett. 85, 1807.

81. Falk, A. and Petrov, A. (2000), Phys. Rev. Lett. 85, 252.

82. Dighe, A., Dunietz, I. and Fleischer, R. (1999), Eur. Phys. J. C6, 647.

83. Dighe, A., Dunietz, I., Lipkin, H. and Rosner, J. (1996), Phys. Lett. B369, 144.

84. Fleischer, R. (1999), Phys. Rev. D60, 073008.

85. Nir, Y. and Silverman, D. (1990), Nucl. Phys. B345, 301.

86. See, for instance, Chang, D. (1983), Nucl. Phys. B214 435;

Ecker, G. and Grimus, W. (1985), Nucl. Phys. B258, 328; (1986), Z. Phys. C30, 293.

87. Ball, P., Frère, J.-M. and Matias, J. (2000), Nucl. Phys. B572, 3.

88. Ball, P. and Fleischer, R. (2000), Phys. Lett. B475, 111.

89. Silverman, D. (1998), Phys. Rev. D58, 095006.

90. CDF Collaboration (Affolder, T. et al.) (2000), FERMILAB-PUB-00-165-E [hepex/0007034].

91. CLEO Collaboration (D. Cronin-Hennessy et al.) (2000), Phys. Rev. Lett. 85, 515 and 525 .

92. Babar Collaboration (Aubert, B. et al.) (2000), SLAC-PUB-8536 [hep-ex/0008057].

93. Belle Collaboration (Abashian, A. et al.) (2000), BELLE-CONF-0005 and 0006.

94. Fleischer, R. and Mannel, T. (1997), TTP-97-22 [hep-ph/9706261];

Choudhury, D., Dutta, B. and Kundu, A. (1999), Phys. Lett. B456, 185;

He, X.-G., Hsueh, C.-L. and Shi, J.-Q. (2000), Phys. Rev. Lett. 84, 18;

Grossman, Y., Neubert, M. and Kagan, A.L. (1999), J. High Energy Phys. 9910, 029.

95. Fleischer, R. and Matias, J. (2000), Phys. Rev. D61, 074004.

96. Fleischer, R. (1996), Phys. Lett. B365, 399;

Gronau, M. and Rosner, J. (1998), Phys. Rev. D57, 6843.

97. Fleischer, R. and Mannel, T. (1998), Phys. Rev. D57, 2752.

98. Fleischer, R. (1999), Eur. Phys. J. C6, 451.

99. Neubert, M. and Rosner, J. (1998), Phys. Lett. B441, 403; Phys. Rev. Lett. 81, 5076 ;

Neubert, M. (1999), J. High Energy Phys. 9902, 014.

100. Buras, A.J. and Fleischer, R. (2000), Eur. Phys. J. C16, 97.

101. Gronau, M., Rosner, J. and London, D. (1994), Phys. Rev. Lett. 73, 21.

102. Hernández, O., London, D., Gronau, M. and Rosner, J. (1994), Phys. Lett. B333, 500; Phys. Rev. D50, 4529; 
Gronau, M., Hernandez, O., London, D. and Rosner, J. (1995), Phys. Rev. D52, 6356. 103. Wolfenstein, L. (1995), Phys. Rev. D52, 537;

Donoghue, J.F., Golowich, E., Petrov, A.A. and Soares, J.M. (1996), Phys. Rev. Lett. 77, 2178;

Buras, A., Fleischer, R. and Mannel, T. (1998), Nucl. Phys. B533, 3;

Neubert, M. (1998), Phys. Lett. B424, 152;

Gérard, J.-M. and Weyers, J. (1999), Eur. Phys. J. C7, 1;

Falk, A., Kagan, A., Nir, Y. and Petrov, A. (1998), Phys. Rev. D57, 4290 (1998);

Atwood, D. and Soni, A. (1998), Phys. Rev. D58, 036005.

104. Fleischer, R. (1998), Phys. Lett. B435, 221;

Gronau, M. and Rosner, J. (1998), Phys. Rev. D58, 113005;

Gronau, M. and Pirjol, D. (1999), Phys. Lett. B449, 321;

Agashe, K. and Deshpande, N. (1999), Phys. Lett. B451, 215 and B454, 359.

105. Hou, W.-S. and Yang, K.-C. (2000), Phys. Rev. D61, 073014.

106. Bander, M., Silverman, D. and Soni, A. (1979), Phys. Rev. Lett. 43, 242.

107. Simma, H. and Wyler, D. (1991), Phys. Lett. B272, 395;

Fleischer, R. (1993), Z. Phys. C58, 483;

Ward, B.F. (1995), in Ref. [47].

108. Chang, C.H. and Li, H.-n. (1997), Phys. Rev. D55, 5577;

Yeh, T.W. and Li, H.-n. (1997), Phys. Rev. D56, 1615;

Keum, Y.Y., Li, H.-n. and Sanda, A.I. (2000), KEK-TH-642 [hep-ph/0004004].

109. Ali, A., Kramer, G. and Lü, C.-D. (1998), Phys. Rev. D58, 094009;

Keum, Y.-Y., Li. H-n., and Sanda, A.I. (2000), NCKU-HEP-00-01A [hep-ph/0004173];

Muta, T., Sugamoto, A., Yang, M.-Z. and Yang, Y.-D. (2000), Phys. Rev. D62, 094020.

110. Skands, P.Z. (2000), hep-ph/0010115.

111. Gronau, M. and Rosner, J. (2000), Phys. Lett. B482, 71;

Chiang, C.-W. and Wolfenstein, L. (2000), hep-ph/0004255.

112. Dunietz, I. (1993), in the proceedings of the Workshop on B Physics at Hadron Accelerators, Snowmass, Colorado, eds. McBride, P. and Shekhar Mishra, C., p. 83;

Lipkin, H. (1997), Phys. Lett. B415, 186;

Buras, A., Fleischer, R. and Mannel, T. (1998), in Ref. [103];

Falk, A., Kagan, A., Nir, Y. and Petrov, A. (1998), in Ref. [103];

Pirjol, D. (1999), Phys. Rev. D60, 054020.

113. Gronau, M. (2000), Phys. Lett. B492, 297.

114. Ali, A. (1997), in the proceedings of the 7th International Symposium on Heavy Flavor Physics, Santa Barbara, California, July 7-11, 1997, ed. Campagnari, C., World Scientific, Singapore, p. 196 [hep-ph/9709507];

Greub, C. (1999), BUTP-99-22 [hep-ph/9911348];

Misiak, M. (2000), hep-ph/0002007. 